NBER WORKING PAPER SERIES

\title{
OPTIMAL INTEREST-RATE RULES: \\ I. GENERAL THEORY
}

Marc P. Giannoni

Michael Woodford

Working Paper 9419

http://www.nber.org/papers/w9419

\author{
NATIONAL BUREAU OF ECONOMIC RESEARCH \\ 1050 Massachusetts Avenue \\ Cambridge, MA 02138 \\ December 2002
}

An earlier version of this work was delivered by the second author as the Jacob Marschak Lecture at the 2001 Far Eastern Meeting of the Econometric Society, Kobe, Japan, July 21, 2001. We thank Ed Nelson, Julio Rotemberg and Lars Svensson for helpful discussions, and the National Science Foundation, through a grant to the NBER, for research support. The views expressed herein are those of the authors and not necessarily those of the National Bureau of Economic Research.

(C) 2002 by Marc P. Giannoni and Michael Woodford. All rights reserved. Short sections of text not to exceed two paragraphs, may be quoted without explicit permission provided that full credit including, $\odot$ notice, is given to the source. 
Optimal Interest-Rate Rules: I. General Theory

Marc P. Giannoni and Michael Woodford

NBER Working Paper No. 9419

December 2002

JEL No. E52, E61

\section{$\underline{\text { ABSTRACT }}$}

This paper proposes a general method for deriving an optimal monetary policy rule in the case of a dynamic linear rational-expectations model and a quadratic objective function for policy. A commitment to a rule of the type proposed results in a determinate equilibrium in which the responses to shocks are optimal. Furthermore, the optimality of the proposed policy rule is independent of the specification of the stochastic disturbances. Finally, the proposed rules can be justified from a "timeless perspective," so that commitment to such a rule need not imply timeinconsistent policy.

We show that under fairly general conditions, optimal policy can be represented by a generalized Taylor rule, in which however the relation between the interest-rate instrument and the other target variables is not purely contemporaneous, as in Taylor's specification. We also offer general conditions under which optimal policy can be represented by a "super-inertial" interest-rate rule, and under which it can be represented by a pure "targeting rule" that makes no explicit reference to the path of the instrument.

Marc P. Giannoni

Graduate School of Business

Columbia University

New York, NY 10027

mg2190@columbia.edu
Michael Woodford

Department of Economics

Princeton University

Princeton, NJ 08544

and NBER

woodford@princeton.edu 
Both positive and normative accounts of monetary policy are often expressed in terms of systematic rules for determining the central bank's operating target for a short-term nominal interest rate. For example, the "Taylor rule" (Taylor, 1993) expresses the Fed's operating target for the federal funds rate as a linear function of a current inflation rate and a current measure of output relative to potential. Alternatively, "inflation targeting" is often described as a commitment to adjust a nominal interest-rate instrument as necessary in order to bring about an inflation projection (say, over a two-year horizon) consistent with the central bank's inflation target (e.g., Vickers, 1998; Svensson, 1999a). A common feature of such prescriptions is that a precise criterion is given that should be checked each time an interest-rate decision is made, in order to determine whether the central bank's current interest-rate target is acceptable or not, given the observed or projected behavior of variables such as inflation and the "output gap" (i.e., the variables that define the bank's stabilization objectives, rather than any "intermediate" targets).

This paper considers the optimal choice of a criterion of this form to guide monetary policy deliberations. The question has been extensively discussed in recent years. ${ }^{1}$ However, most of the recent literature assumes some low-dimensional parametric family of policy rules, and then optimizes over the coefficients of the rule, using an economic model to compute the equilibrium associated with each possible set of parameters. A characteristic weakness of such work, ${ }^{2}$ in our view, is that the conclusions reached about the optimal values of certain parameters are likely to be strongly influenced by the parametric family of rules considered, i.e., by which other kinds of feedback are assumed not to be possible. Hence we propose here to take a different approach. We propose first to characterize the best possible pattern of equilibrium responses to disturbances - solving an optimization problem in which the structural equations of one's model of the economy appear as constraints - and then ask what kind of policy rule can bring about the desired equilibrium.

This alternative approach is standard in the theory of public finance, and is also used in

\footnotetext{
${ }^{1}$ See, e.g., the papers in Taylor (1999).

${ }^{2}$ This includes our own previous studies, such as Rotemberg and Woodford (1999).
} 
optimal-control approaches to the analysis of monetary policy, such as that of Currie and Levine (1993). However, work of this latter kind typically assumes that optimal policy has been adequately characterized once one has solved for the optimal instrument setting as a function of the history of exogenous disturbances. ${ }^{3}$ Instead, our primary interest will be in the further question of how best to choose a policy rule with which to implement the optimal pattern of responses to disturbances. We shall argue that a policy rule specified in terms of a mapping from the history of disturbances to the instrument setting is not the only possible type of policy rule that would be consistent with the optimal pattern of responses, and that it is generally not the best one. Thus our concern with policy rules does not derive from the idea we should be willing to accept an outcome that is not as good as what could be achieved by a more sophisticated policy, purely for the sake of maintaining a simple policy commitment. Instead, we note that many forms of policy commitment are equally suitable for implementing the optimal equilibrium, so that we can ask for a representation of optimal policy that has other desirable features as well.

For example, we shall demand that our policy rule be not merely consistent with the desired equilibrium, but also that commitment to the rule imply a determinate equilibrium, so that the rule is not equally consistent with other, less desirable equilibria. We shall also look for policy rules that are time-invariant, and that refer only to the evolution of certain state variables (those that we call target variables) that represent the central bank's stabilization goals. Finally, we shall seek to derive policy rules that continue to be optimal regardless of what the statistical properties of the exogenous disturbances hitting the economy are believed to be.

In this paper, we present a method for deriving policy rules with these properties, for a fairly general class of policy problems in which the monetary transmission mechanism is represented by a linear(ized) rational-expectations model, and stabilization objectives are represented by a discounted quadratic loss function. ${ }^{4}$ Under fairly general circumstances,

\footnotetext{
${ }^{3}$ These literatures also typically identify optimal policy with what we call a " $t_{0}$-optimal commitment" in section 3 below, whereas we argue for a slightly different concept of optimal policy, that does not seek to exploit the expectations that people happen to have held prior to the adoption of the policy.
} 
optimal policy can be represented by a sort of generalized Taylor rule, i.e., an expression for the central bank's operating target as a linear function of the projected paths of the target variables. However, an optimal criterion of this kind is generally not a purely contemporaneous one, as in Taylor's (1993) rule. We show that the presence of forward-looking terms in the model's structural equations necessarily implies history-dependence of the optimal policy rule; in particular, the optimal instrument setting is a function of lagged values of the instrument, even if only the contemporaneous value of the instrument enters the structural equations. We furthermore offer general conditions under which an optimal instrument rule will involve super-inertial dynamics for the policy instrument, as in the optimal interest-rate rules derived by Rotemberg and Woodford $(1997,1999)$. We also show, conversely, that the presence of lagged endogenous variables in the model's structural equations implies that an optimal policy rule will be at least somewhat forward-looking. ${ }^{5}$

We also find that in general, an optimal rule of the kind proposed here defines the central bank's operating target only implicitly; that is, the central bank cannot solve for the appropriate instrument setting without projecting the paths of variables that depend on the setting that it chooses. Thus the central bank must use a model of the economy in choosing the level of interest rates, as in the inflation-forecast targeting regimes mentioned above. Indeed, our optimal rules can be viewed as "targeting rules" in the sense of Svensson (1997, 1999a, 2001) and Svensson and Woodford (1999) — rules that are specified in terms of a criterion that certain target variables are to satisfy as a result of the way the central bank adjusts its instrument - even when they involve an implicit expression for the interest rate, and in some cases they can only be understood as "targeting rules".

In sections 1-4, we discuss further our general criteria for an optimal policy rule, and state them more precisely. We then offer in sections 5-7 a general approach to the derivation of optimal policy rules that allows us to derive a rule (or rules) satisfying all of these criteria

\footnotetext{
${ }^{4}$ Conditions under which the linear policies that are optimal for a problem of this kind represent a linear approximation to optimal policy from the point of view of expected-utility maximization in an intertemporal general-equilibrium model are presented in Woodford (2002, chap. 6).

${ }^{5}$ The degree to which an optimal rule is likely to be forward-looking in practice is considered quantitatively in our companion paper, Giannoni and Woodford (2002).
} 
in the case of a fairly general linear-quadratic policy problem. In this general discussion, the instrument of policy need not be an interest rate, and the stabilization goals need have nothing to do with nominal variables, though this is the application that motivates our formulation of the problem. Applications to the problem of interest-rate policy in the context of explicit optimizing models of the monetary transmission mechanism are then taken up in a companion paper (Giannoni and Woodford, 2002).

\section{Alternative Forms of Policy Rules}

We begin by discussing in general terms the properties that we would like a monetary policy rule to have, in order for it to be considered a suitable approach to implementing the optimal responses to disturbances that one can characterize using standard controltheoretic methods. A first issue is what we should consider to be a complete specification of a monetary policy rule. This might seem to have a simple answer: a policy rule should specify the central bank's instrument setting as a function of the "state of the world" at that time, which would be identified by the complete history of exogenous disturbances up through the current date. But we shall not insist upon this sort of representation of optimal policy. First, it is not the way in which the sorts of policy commitments that are actually discussed in central-banking circles are ever expressed. Instead, popular current proposals, such as the Taylor rule (Taylor, 1993) or inflation-forecast targeting (Svensson, 1999a), specify responses to the actual and/or projected evolution of endogenous variables such as inflation and real activity. That such proposals appear more practical (whatever their other merits) is clear; a rule specified in terms of the appropriate response to "this quarter's technology shock" and so on would obviously raise questions about the measurement of the state in question that are even more severe than the questions that arise in practice about the appropriate measurement of inflation and of the "output gap". An even more serious problem is the fact that it would not be feasible, in practice, to list all of the possible types of real disturbances on which optimal state-contingent policy should depend; thus identification of the state of the economy through a complete enumeration of the exogenous disturbances (let alone their 
complete histories!) is not really possible for an actual economy. Of course, description of the economy's state in terms of a small number of endogenous variables may or may not be an adequate substitute, but it is clear that we should consider the possible advantages of rules specified in that way rather than excluding them a priori. In fact, we show below that certain other desiderata - such as commitment to a policy rule that implies a determinate equilibrium - may require that the policy rule specify feedback from endogenous variables.

It is for this reason that the question of how to implement optimal policy remains a non-trivial one, even after the optimal responses to shocks have been computed. And once we admit the possibility of rules that specify feedback from endogenous variables, it is rather obvious that there will cease to be a uniquely optimal policy rule; for the same pattern of equilibrium adjustments of the policy instrument may be equivalently described in terms of feedback from any of a variety of endogenous variables that all respond to the same ultimate disturbances in equilibrium. This is especially true if our model assumes the existence of only a few real disturbances, and less so if there are as many distinct disturbances as endogenous variables. But even in the latter case, there will be a large multiplicity of rule specifications that are equally consistent with the optimal equilibrium, if we allow the rule to specify feedback from exogenous as well as endogenous variables. And while the additional requirement that the rule imply a determinate equilibrium reduces the set of possibilities, this generally amounts to requiring that the coefficients of the feedback rule satisfy certain inequalities, that will not suffice to determine unique coefficients. ${ }^{6}$ It is for this reason that we are able to ask for rules with other desirable properties as well, such as the robustness that we discuss below.

The policy problems that we treat in this paper are ones in which the constraints upon the feasible set of paths for the various state variables can be represented by a system of linear (or log-linear) equations, and in which the policymaker's objective can be represented by a quadratic function of these variables. In such cases, optimal policy rules can be described by linear relations among the state variables. Thus the general form of policy rule that we

\footnotetext{
${ }^{6}$ See, e.g., Svensson and Woodford (1999) and Woodford (2002, chap. 4).
} 
wish to consider is a relation of the form

$$
\phi_{i} i_{t}+\phi_{z}^{\prime} \bar{z}_{t}+\phi_{Z}^{\prime} \bar{Z}_{t}+\phi_{s}^{\prime} \bar{s}_{t}=\bar{\phi}
$$

where $i_{t}$ is the policy instrument (assumed for simplicity to be a scalar ${ }^{7}$ ), the value of which is chosen in period $t, \bar{z}_{t}$ is a vector of non-predetermined endogenous variables ("jump variables"), the values of which may depend upon both the policy action and exogenous disturbances at date $t, \bar{Z}_{t}$ is a vector of predetermined endogenous variables (lags of variables that are included in $\bar{z}_{t}$ ), $\bar{s}_{t}$ is a vector of exogenous state variables (disturbances) that affect the equilibrium evolution of the endogenous variables, and the coefficients $\phi_{i}, \phi_{z}$, and so on are constants. This notation should be understood to allow dependence of current policy both on forecasts (for example, the inflation forecast $\mathrm{E}_{t} \pi_{t+k}$ may be an element of $\bar{z}_{t}$ ) and on past policy (as $i_{t-j}$ may be an element of $\bar{Z}_{t}$ ). Our assumption here that the intercept term $\bar{\phi}$ is not time-varying reflects a time-invariance property that we argue below to be a desirable feature of a policy rule.

We assume that either $\phi_{i} \neq 0, \phi_{z} \neq 0$, or both, so that (1.1) constrains possible endogenous outcomes at date $t$, rather than referring only to variables that cannot be affected by the policy decision at date $t$. In the case that $\phi_{i} \neq 0$, but $\phi_{z}=0$, equation (1.1) can be solved for the instrument setting $i_{t}$ as an explicit function of predetermined and exogenous state variables alone, which variables have determinate values independent of the policy decision. In this case the rule is what Svensson and Woodford (1999) call an explicit instrument rule. One might suppose that a fully specified policy rule should be explicit in this sense. However, in this case some popular practical proposals such as inflation-forecast targeting (Svensson, 1999a) would be excluded as unsuitable in form. Yet the use of such rules as guides to policy in central banks suggests that it is of interest to consider the possibility of representing optimal policy in such a form.

Furthermore, we shall show that desirable policy rules (on grounds to be discussed below) can typically not be expressed in this form. While these rules could equivalently be expressed

\footnotetext{
${ }^{7}$ It should be obvious in what follows that our equations have a natural extension to the case in which $i_{t}$ represents a vector of instruments the values of which are chosen each period.
} 
as explicit instrument rules — by substituting for current inflation the equilibrium value of that variable as a function of predetermined and exogenous state variables, and similarly for other variables, including the current inflation forecast - this explicit description would not remain the same in the case of a change in the specified properties of the exogenous disturbance processes. Thus the explicit version of the rule would lack the robustness of the version that we shall prefer. For this reason it is desirable to consider rules that implicitly specify an instrument setting at each point in time, even if the central bank has to use a complete model of the economy to determine the actual instrument setting, rather than being able to delegate the task of policy implementation to a clerk with a calculator as in the case of a rule that is "operational" in McCallum's (1999) sense.

It is arguable that "operationality" is desirable, on the ground that in this case can the central bank's fulfillment of its supposed commitments be directly verified by members of the public. ${ }^{8}$ But while we note that public understanding of and confidence in the central bank's commitments is crucial to successful stabilization policy, it is not clear that the improvement in the private sector's ability to predict central bank policy as a result of commitment to a rigid, simple rule should be great enough to outweigh the advantages of a more flexible specification (should the latter policy still be fairly well understood). In any event, it seems unlikely under present circumstances, as Svensson (2001) has stressed, that any central bank would be willing to commit itself to an explicit, mechanical rule of this kind, and so we shall not limit our study to consideration of rules of that kind.

An implicit policy rule in which both $\phi_{i}$ and $\phi_{z}$ are non-zero is an implicit instrument

\footnotetext{
${ }^{8}$ If this is the ground for insistence upon the requirement, then one should also require that the rule specify feedback only from state variables corresponding to published statistics, rather than allowing dependence upon exogenous disturbances. It is clear that not all systematic policies need have an equivalent representation of this form; for example, such a rule cannot allow the period $t$ nominal interest rate to depend, either directly or indirectly, upon any disturbances to the economy in period $t$. Thus the study of what can be achieved by rules that are "operational" in such a strict sense requires one to consider the degree to which optimal policy can be approximated by a simple rule that is admittedly not fully optimal (but may be preferred for its simplicity). This topic is not treated here, as it cannot usefully be addressed except in the context of a model that is intended as a realistic full representation of the various types of disturbances affecting the economy. Rotemberg and Woodford (1999) provides an example of such a study, in which rules that are operational in McCallum's sense are among the families of simple rules considered.
} 
rule. This is a formula for setting the policy instrument as a function of other variables, some of which must be projected by the central bank in order to implement the rule, with the projections themselves being conditional upon (and affected by) the instrument setting. Taylor's classic (1993) formulation of his rule is arguably of this kind, since the quarter $t$ federal funds rate target is specified as a function of the quarter $t$ GDP deflator and quarter $t$ real GDP, both of which would have to be projected at the time that the funds rate is set. In the case of a bank with a model of the economy that implies that current-quarter inflation or output should depend on current-quarter interest rates, such a rule is an implicit instrument rule. ${ }^{9}$ Other examples would include the rules, specifying the short-term nominal interest rate as a function of an inflation forecast, that are sometimes used to represent the policies of inflation-targeting central banks (e.g., Black et al., 1997; Batini and Haldane, 1999).

Of course, given that such rules specify the instrument setting only implicitly, an obvious question arises as to whether they represent a well-defined policy specification at all. Our view is that they do if and only if the rule in question, when adjoined to the other equations of a structural model, is consistent with the existence of a rational-expectations equilibrium, and implies a determinate solution for the state-contingent path of the policy instrument (in the sense described further in the next section). This means that the question whether a given implicit rule can be considered a well-defined policy specification is model-dependent. However, it depends only upon the coefficients by which the endogenous variables enter the (linear) structural equations of one's model, and not upon the assumed properties of the exogenous disturbance processes. We argue below that robustness to changes in beliefs about the nature of the exogenous disturbances is the primary sense in which it is important for a proposed policy rule to be robust.

Relation (1.1) can also describe a well-defined policy rule in the case that $\phi_{i}=0$, as long as $\phi_{z} \neq 0$. In this case, the equation must be understood to specify a (pure) targeting rule

\footnotetext{
${ }^{9}$ On the other hand, if inflation and output are both predetermined variables, as in many models, then the Taylor rule would be an example of an explicit instrument rule. Optimizing models of this kind are discussed in Giannoni and Woodford (2002).
} 
of the kind advocated by Svensson (1999a, 2001). ${ }^{10}$ Such a rule specifies a target criterion that the central bank's projection of the economy's future path should satisfy; the bank is committed each period to adjust its instrument as necessary to bring about a condition in which its projection satisfies the target criterion. An example (Svensson's "strict inflation targeting" rule) would be the criterion that the conditional forecast at date $t$ of inflation at some date $t+k$ always equal the inflation target $\bar{\pi}$; in a more complicated example (that Svensson offers as an example of "flexible inflation targeting"), the criterion involves both the conditional forecast of future inflation and a conditional forecast of the output gap, possibly at a shorter horizon. In neither case does the criterion that the central bank commits to maintain directly involve its instrument. However, such a rule may represent a feasible and complete specification of policy, just as with an implicit rule that provides a formula for the instrument setting. Once again, we shall consider that the rule represents a well-defined policy specification if, when adjoined to the other structural equations, it is consistent with the existence of a rational-expectations equilibrium and implies a determinate solution for the path of the instrument. Once we admit that implicit rules can represent well-defined policies, there is really no reason to restrict attention to rules that are expressed as formulas for the instrument setting, and indeed the distinction between instrument rules and targeting rules is probably not as important as that between explicit rules and implicit rules (of either type). ${ }^{11}$

Finally, we consider whether it is desirable to restrict our attention to policy rules that are purely forward-looking in the sense of Woodford (2000). These are rules (whether explicit or implicit) that make policy a function only of state variables that are relevant to the determination of current or future target variables (i.e., variables that affect the central bank's loss function either currently or in the future). In the kind of policy problem considered

\footnotetext{
${ }^{10}$ Svensson further distinguishes between "general" and "specific" targeting rules; here we consider only the latter way of specifying a policy commitment. See also Svensson and Woodford (1999).

${ }^{11}$ Note that implicit instrument rules might also be considered to be examples of targeting rules, in which the target criterion happens to involve the central bank's instrument; for example, Svensson (2001) discusses targeting rules that involve a short-term nominal interest rate. We shall speak of a pure targeting rule in the case that the targeting rule cannot alternatively be interpreted as an implicit instrument rule.
} 
here, we assume a discounted quadratic loss function of the form

$$
\mathrm{E}_{t_{0}} \sum_{t=t_{0}}^{\infty} \beta^{t-t_{0}} L_{t}
$$

where $t_{0}$ is the initial date at which a policy rule is adopted, $0<\beta<1$ is a discount factor, and the period loss $L_{t}$ is of the form

$$
L_{t}=\frac{1}{2}\left(\tau_{t}-\tau^{*}\right)^{\prime} W\left(\tau_{t}-\tau^{*}\right)
$$

where $\tau_{t}$ is a vector of target variables, $\tau^{*}$ specifies the vector of target values for these variables, and $W$ is a symmetric, positive-definite matrix. The target variables are furthermore assumed to be linear functions of a subset of the endogenous variables mentioned earlier,

$$
\tau_{t}=T y_{t}
$$

where

$$
y_{t} \equiv\left[\begin{array}{c}
Z_{t} \\
z_{t} \\
i_{t}
\end{array}\right],
$$

$Z_{t}$ is a subset of the vector of predetermined variables $\bar{Z}_{t}, z_{t}$ is a subset of the vector of nonpredetermined endogenous variables $\bar{z}_{t}$, and $T$ is a matrix of coefficients. We furthermore suppose that $Z_{t}$ includes all of the predetermined endogenous variables that constrain the possible equilibrium evolution of the variables $Z_{T}$ and $z_{T}$ for $T \geq t$, and that a subset $s_{t}$ of the exogenous states includes all of the exogenous states that contain information about the possible future evolution of the variables $Z_{T}$ and $z_{T}$ for $T \geq t$.

A rule (1.1) is then purely forward-looking if it involves non-zero weights on variables in $\bar{z}_{t}, \bar{Z}_{t}$, or $\bar{s}_{t}$ only to the extent that (i) they have non-zero weights in (1.4), (ii) they enter with non-zero weights in the structural equations that determine the possible values of variables $z_{t}$ of type (i), (iii) lagged values of these variables are elements of $Z_{t}$ or $s_{t}$ of types (i) or (ii), so that the current values are relevant to the determination of target variables at some later date, or (iv) they represent conditional expectations at date $t$ of variables of types (i) or (ii) at some later date. It follows that a purely forward-looking rule will involve only the 
instrument $i_{t}$ and the variables in $z_{t}, Z_{t}$, and $s_{t}$, together (perhaps) with various conditional expectations of the form $\mathrm{E}_{t} z_{t+j}$.

In the case of the loss function assumed in the examples in Giannoni and Woodford (2002), the target variables are inflation, the output gap, and the short-term nominal interest rate itself. Thus examples of purely forward-looking rules would include implicit instrument rules that specify the short-term nominal interest rate as a function solely of projections of current and future values of inflation and the output gap. They would also include targeting rules in which the target criterion involves only projections of current or future inflation rates and output gaps. Many popular proposals are obviously of this kind, though the stress on the forward-looking character of recommended policy has probably been greatest in the literature on inflation-forecast targeting.

One might suppose on dynamic-programming grounds that optimal policy should have no need for dependence on state variables that enter neither the objective function nor the constraints of the policy problem looking forward from a given date, and thus that there would be no loss of generality in restricting our attention to purely forward-looking rules. Doing so might be expected to have the advantage of eliminating redundant complexity from our representation of optimal policy, and thus to reduce the multiplicity of optimal rules that would otherwise exist.

Unfortunately, as explained in Woodford (1999a), this "dynamic programming" argument is incorrect in the case of forward-looking private-sector behavior. The optimal statecontingent path of the central bank's instrument generally involves additional history-dependence; it is optimal for the bank to commit itself to condition its later policy upon disturbances that no longer constrain the possible evolution of the target variables, because of the effect of the anticipation of such dependence upon private-sector behavior at earlier dates. A purely forward-looking policy rule can at best be chosen to implement what Woodford (1999a) calls "the optimal non-inertial plan"; but as is shown there, this is generally not fully optimal. Thus, while we do give brief consideration in Giannoni and Woodford (2002) to optimal policy within this restricted class of rules, we do not limit our attention to rules of this kind. 
Finally, we shall give particular attention to policy rules that are simple in a different respect. By a direct instrument rule we shall mean a rule that specifies feedback only from the observed and/or projected paths of the target variables, and from the past evolution of the instrument itself, if this is not one of the target variables. Such a rule thus specifies a direct connection between the bank's ultimate objectives, the target variables $\tau_{t}$, and the variable about which it makes an immediate decision, its instrument $i_{t}$, that does not involve any "intermediate target" variables. We shall similarly define a direct targeting rule as one in which the target criterion involves only (leads and lags of) the target variables.

It is obvious that many popular current proposals, including both the Taylor rule and standard formulations of inflation-forecast targeting, are direct rules in this sense. Direct rules evidently have a degree of practical appeal, and probably facilitate communication with the public about the nature of policy as well. Reference to ambiguous state variables such as "the output gap" obviously presents some difficulties, both for the implementation of a policy rule and for the explanation of policy to the public; but if such terms of art must be used - and in general they must be, if the policy rule is to be robustly optimal - it is probably best to use terms that refer directly to the goals of policy, so that the meaning of the variable can be discussed in terms of what a desirable target (say, for output) is believed to be, rather than terms that have no meaning except in the context of a particular model of the economy.

We shall find that it is possible to formulate direct rules that are optimal, and indeed robustly optimal, in a sense that is defined in section 4 below. We could formulate a large class of alternative rules that would also be robustly optimal (in the same sense), by substituting for one or another variable in terms of others using one or more of the structural equations of our model of the economy. However, these alternative representations of optimal policy would all involve additional variables, such as the exogenous disturbances, that enter the structural equations of the model. Restricting attention to optimal direct rules allows us to reach much more definite conclusions about the nature of an optimal rule, and these are the results that we shall emphasize. 


\section{Determinacy of Equilibrium}

We turn now to a more precise discussion of the desiderata that we would like a policy rule to satisfy. First of all, we shall consider only rules that imply a determinate rational expectations equilibrium, by which we mean a unique equilibrium with the property that bounded disturbance processes result in bounded fluctuations in the endogenous variables. ${ }^{12}$ Rules that imply indeterminacy are problematic for several reasons. As noted above, in the case of an implicit rule, it is not even clear that an equation of the form (1.1) specifies any well-defined policy.

And even in the case of an explicit instrument rule, a rule that implies indeterminacy is clearly undesirable. The mere fact that such a rule may be consistent with a desirable equilibrium is little recommendation for the rule if it is also equally consistent with other, much less desirable equilibria. In the case of the linear models and quadratic loss functions considered here, that is necessarily the case. A rule that implies indeterminacy will be consistent with a large set of equilibria, including ones in which the fluctuations in endogenous variables are arbitrarily large relative to the size of fluctuations in the exogenous disturbances (Woodford, 2002, chap. 4). Except in non-generic special cases, the variables in which there may be arbitrarily large fluctuations due to self-fulfilling expectations will include the target variables. Hence at least some of the equilibria consistent with the rule will be less desirable, in terms of the loss function specified by $(1.2)-(1.3)$, than the unique bounded equilibrium associated with any rule with a determinate equilibrium.

To be more specific, let us assume that the evolution of the endogenous variables $z_{t}$ and $Z_{t}$ that are relevant to the central bank's stabilization objectives is determined by a system

\footnotetext{
${ }^{12}$ The meaning of a criterion of boundedness depends, of course, upon which variables one uses to describe the equilibrium in question; in a given equilibrium, the inflation rate may be a bounded process while the log price level is not. We shall not be concerned by unboundedness of variables that are linked to the bounded variables in the model only by relations that hold exactly, as in the case of the identity $\pi_{t}=\log P_{t}-\log P_{t-1}$. In such a case, the existence of unbounded fluctuations in the log price level does not imply any unbounded approximation associated with a linear approximation to the model's structural relations.
} 
of simultaneous equations

$$
\hat{I}\left[\begin{array}{c}
Z_{t+1} \\
\mathrm{E}_{t} z_{t+1}
\end{array}\right]=A\left[\begin{array}{c}
Z_{t} \\
z_{t}
\end{array}\right]+B i_{t}+C s_{t}
$$

Each matrix in this equation has $n=n_{z}+n_{Z}$ rows, where $n_{z}$ is the number of nonpredetermined and $n_{Z}$ is the number of predetermined endogenous variables, ${ }^{13}$ so that there are a sufficient number of independent structural relations each period to determine each of the endogenous variables. We may further partition the matrices as

$$
\hat{I}=\left[\begin{array}{cc}
I & 0 \\
0 & \tilde{E}
\end{array}\right], \quad A=\left[\begin{array}{cc}
A_{11} & A_{12} \\
A_{21} & A_{22}
\end{array}\right], \quad B=\left[\begin{array}{c}
0 \\
B_{2}
\end{array}\right], \quad C=\left[\begin{array}{c}
0 \\
C_{2}
\end{array}\right]
$$

where in each case the upper blocks have $n_{Z}$ rows and the lower blocks $n_{z}$ rows, and the columns of $\hat{I}$ and $A$ are partitioned in a manner conformable to the partition of the endogenous variables in (2.1). Here the assumed zero restrictions in the upper blocks reflect the fact that the first $n_{Z}$ equations define the elements of $Z_{t}$ as elements of $z_{t-j}$ for some $j \geq 1$. (Because of this feature of the vector $Z_{t+1}$, the assumption that the lower left block of $\hat{I}$ is a zero matrix is also without loss of generality.) We assume that $B_{2}$ is not zero in all elements, so that the instrument has some effect, and that $A_{22}$ is non-singular, so that the last $n_{z}$ equations can be solved for $z_{t}$ as a function of $Z_{t}, s_{t}, i_{t}$, and expectations $\mathrm{E}_{t} z_{t+1}$.

Insofar as the policy rule (1.1) involves non-zero coefficients on elements of $\bar{z}_{t}$ or $\bar{Z}_{t}$ not included in the sub-vectors $z_{t}$ and $Z_{t}$, it is necessary to augment the equilibrium conditions (2.1) with the additional conditions that determine the equilibrium evolution of the additional endogenous variables. We shall suppose that the augmented system of equilibrium conditions can again be written in the form

$$
\bar{I}\left[\begin{array}{c}
\bar{Z}_{t+1} \\
\mathrm{E}_{t} \bar{z}_{t+1}
\end{array}\right]=\bar{A}\left[\begin{array}{c}
\bar{Z}_{t} \\
\bar{z}_{t}
\end{array}\right]+\bar{B} i_{t}+\bar{C} \bar{s}_{t},
$$

\footnotetext{
${ }^{13}$ We assume that both $z_{t}$ and $Z_{t}$ are vectors of finite length. The vector $s_{t}$, however, need not be; as we discuss in section 4 below, we allow in principle for an infinite number of distinct types of random disturbances. In the case that $s_{t}$ is not a finite vector, our references to "bounded disturbance processes" are to be understood to refer not simply to a bound upon each element of $s_{t}$, but also to a bound upon each element of $C s_{t}$, so that the perturbations of the structural relations are bounded (and well-defined) each period.
} 
where the larger matrices $\bar{I}$ and $\bar{A}$ contain $\hat{I}$ and $A$ respectively as diagonal blocks, the vector $\bar{B}$ contains $B$ as a sub-vector, and so on.

We are here concerned solely with bounded solutions to these equations, i.e., with solutions in which each element of $\bar{z}_{t}$ and $\bar{Z}_{t}$ satisfies some bound for all $t$, under the assumption that the disturbances are bounded (each element of $\bar{C} \bar{s}_{t}$ satisfies some bound for all $t$ ). In the case of an exact linear-quadratic model, one might want to consider unbounded solutions, subject perhaps to a transversality condition, or some other particular bound with an economic interpretation. But in general (as in the applications treated in Giannoni and Woodford, 2002), the structural equations (2.2) will be only linear approximations to a set of true, nonlinear equilibrium conditions, and there will be reason to doubt whether unbounded solutions correspond to any solutions at all of the true equations. We shall accordingly here consider only the set of bounded solutions consistent with a given policy rule, and say that equilibrium is determinate when there exists a unique solution of this kind.

Definition. A policy rule (1.1) implies a determinate rational-expectations equilibrium if the system of equations obtained by conjoining this equation to the system (2.2) has a unique bounded solution for the endogenous variables in periods $t \geq t_{0}$, given the initial conditions $\bar{Z}_{t_{0}}$ and bounded disturbance processes for all periods $t \geq t_{0}$.

A special case of particular interest is that of a policy rule that specifies an instrument setting as a function of the history of exogenous disturbances. (This is often taken to be a natural way of specifying a policy rule, especially in the literature that seeks to characterize optimal commitment.) In this case, the complete system of equilibrium conditions is simply (2.1), with $i_{t}$ replaced by a specified function of $\bar{s}_{t}$. Standard results then imply that the determinacy of equilibrium depends, in the generic case, on the roots of the characteristic equation associated with this system,

$$
\operatorname{det}[A-\mu \hat{I}]=0
$$

Rational-expectations equilibrium is (generically) determinate if the number of roots $\mu_{i}$ such 
that $\left|\mu_{i}\right|<1$ is exactly equal to $n_{Z}$, the number of predetermined state variables.

In the applications that we consider in Giannoni and Woodford (2002), the structural equations are such that this polynomial has more than $n_{Z}$ roots inside the unit circle, and so a policy rule of this kind leads to indeterminacy. This is the Sargent-Wallace (1975) problem with rules that specify an exogenous path for the nominal interest rate, discussed further in Woodford (2002, chap. 4). Hence we propose the following terminology.

Definition. A system (2.1) has the Sargent-Wallace property if (2.3) has more than $n_{Z}$ roots $\mu_{i}$ such that $\left|\mu_{i}\right|<1$, where if $A$ is singular, the zero root is counted $n-\operatorname{rank}(A)$ times.

In such a case, it is important to consider as well the possibility of rules that prescribe feedback from the actual and/or projected paths of endogenous variables.

\section{Optimality from a Timeless Perspective}

Next, we would like for the equilibrium determined by a policy rule to be consistent, in a certain sense, with the optimal commitment that one can characterize using control-theoretic methods (see, e.g., Woodford, 1999b). But the exact sense in which the two should correspond deserves further discussion. Issues of some subtlety arise owing to the failure of the optimal commitment to be time-consistent. If we compute the optimal commitment regarding policy from some date $t_{0}$ forward - which is optimal in the sense of minimizing expected discounted losses from that time onward, conditional upon the state of the economy at that time - this optimal plan will involve behavior that is not independent of the date $t$, i.e., the date relative to the time at which the commitment is chosen. This is because the optimal commitment takes no account of any effect of outcomes in the early periods of the commitment upon expectations prior to the date at which the new policy takes effect (that are simply given when the policy is chosen), whereas the commitment regarding later periods does take account of the effect of predictable outcomes in those periods upon expectations 
in earlier periods (that are nonetheless subsequent to the choice of the policy). As a result it may, for example, be optimal to commit at date $t_{0}$ to a policy that involves inflation immediately following the new commitment, given that this inflation will be unexpected, but that promises no inflation (on average) in the long run.

One may doubt, however, whether it is really desirable for a central bank to commit itself, once and for all, to a time-dependent policy rule intended to implement this time-dependent optimal plan. The problem is not simply that a time-dependent rule is more complex, and thus more difficult to explain both within the bank and to the public. A deeper problem arises from the fact that the time-dependent character of the rule makes it obvious that the rule privileges a particular date, the date at which the policy happens to have been chosen, and that one continues to be committed to a rule that was optimal from the point of view of society's interest at that time, but that would not similarly be chosen today. This raises the obvious question, "What is so special about date zero?" (Svensson, 1999b) — why should one care about following a policy that was optimal from the standpoint of that date rather than any other?

One might answer that once a central bank realizes the value of commitment, it should accept that it is important that it keep its promises, whichever promises those happen to be and whenever they happen to have been announced, simply because they were announced. The problem with this view is that a central bank surely will not be willing to genuinely tie its hands forever; it will not be willing to make a commitment that must be understood in this way, because of the likelihood of unexpected developments that change its view of the nature of optimal policy. The policy rule that seems optimal at one point in time, no matter how great the expertise that has gone into its calculation, will surely seem less ideal as the economy changes and as further experience increases understanding; it may even be seen, at a later date, to be disastrous. No central bank would be willing to forswear attempts to improve upon policy in the light of such increased knowledge. But if the commitment is understood only to bind until the bank's model of the economy changes, at which time a new "optimal commitment" may be chosen on similar grounds, it is hard to see how such 
an approach would not reduce to pure discretion, given the constant availability of grounds for small improvements in one's model.

A possible solution to this dilemma is for the bank to choose a policy rule that implements an equilibrium that is optimal not in the above sense, but from what Woodford (1999b; 2002, chap. 7) calls a "timeless perspective". This means that the rational-expectations equilibrium that is expected to prevail from time $t=t_{0}$ onward, given a commitment to the policy rule at that date, need not minimize expected losses from that date onward, conditional upon the economy's state at that time. Instead, it suffices that the equilibrium be optimal subject to certain constraints on the economy's initial evolution, that prevent the central bank from exploiting the already existing expectations at the time that the policy commitment is chosen. ${ }^{14}$ It is reasonable for the bank to accept these constraints, insofar as they are ones to which it would wish to commit itself in the future, given that, in choosing its commitments for the future, it takes into account the consequences of its commitments for private-sector expectations at earlier dates. Through the same reasoning by which the bank recognizes that it would wish to constrain itself this way in the future (and wish for the private sector to understand it to be constrained), it should recognize that it would have been desirable in the past for it to constrain itself to fulfill these constraints at the present time.

Of course, one may ask why a bank should regard itself as constrained to fulfill a plan to which it would have been optimal for the bank to have committed itself in the past whether or not it did actually announce any such rule in the past, whether or not the private sector did actually expect such a policy in the past, and indeed whether or not the bank's previous understanding of the economy agreed with the model now used to justify the policy

\footnotetext{
${ }^{14}$ Constraints of this kind on initial behavior are often assumed in discussions of dynamic optimal fiscal policy. For example, a constraint on the degree to which initially existing capital can be taxed is introduced to avoid obtaining the result that an optimal policy will involve complete confiscation of initially existing capital, together with a promise never to treat capital similarly in the future; or a constraint on the initial price level is introduced to avoid the result that an optimal policy will involve inflating away the value of initially existing nominal government liabilities, while promising a low rate of inflation thereafter. See McCallum and Nelson (2000) for further discussion of the extent to which a desire for rules that are optimal from a timeless perspective has been implicit in previous discussions both of monetary policy rules and of optimal tax policy.
} 
rule in question as optimal. The answer is that behaving in such a way allows the bank's behavior to be consistent over time, with the advantages that this allows for the credibility to the private sector of the bank's supposed commitments, and for the likelihood that the private sector can learn to predict future policy accurately. The choice of a rule of conduct from such a perspective eliminates the problem of the time inconsistency of optimal policy, in the sense that the same reasoning that is used to support the choice of the optimal rule at one date can be used to support continued adherence to the same rule at any later date (assuming that the central bank's model of the economy has not changed in the meantime). ${ }^{15}$

The selection of a policy rule from this perspective means that it is not necessary to view the choice of the rule as a once-and-for-all commitment, by which the central bank will be bound no matter how unappealing the rule may come to appear at a later time. Instead, the bank need only be committed to determine policy at all later dates by a rule that is optimal from a similarly timeless perspective. Insofar as the bank's model of the economy is expected to guide its decisions in the future as well, there is no reason to expect future behavior that does not conform to the rule currently adopted - and so there is no inconsistency involved in adopting the rule now because of its desirable properties if the bank is expected to follow it indefinitely. At the same time, rule-based policymaking of this sort allows the bank to follow at all times a rule that can be justified as optimal given its best current understanding of the structure of the economy, rather than being constrained by the straightjacket of its past views.

Formally, optimality of a rule from a timeless perspective can be defined as follows.

Definition. A policy rule that determines a unique non-explosive rational-expectations equilibrium is optimal from a timeless perspective if the equilibrium determined by the rule is such that

\footnotetext{
${ }^{15}$ Note that we distinguish here between "time inconsistency" and the existence of an incentive to deviate from the policy previously adopted, should the policymaker not regard itself as constrained not to exploit prior expectations. This follows from the fact that we do not define "optimal policy" as policy that is optimal from the standpoint of the policymaker's current situation, in the absence of any constraints other than those implied by predetermined state variables.
} 
(i) the non-predetermined endogenous variables $z_{t}$ can be expressed as a time-invariant function of a vector of predetermined variables $\bar{Z}_{t}$ and a vector of exogenous variables $\bar{s}_{t}$; that is, a relation of the form

$$
z_{t}=f_{0}+f_{Z} \bar{Z}_{t}+f_{s} \bar{s}_{t}
$$

applies for all dates $t \geq t_{0}$; and

(ii) the equilibrium evolution of the endogenous variables $\left\{y_{t}\right\}$ for all dates $t \geq t_{0}$ minimizes (1.2) among the set of all bounded processes, ${ }^{16}$ subject to the constraints implied by the economy's initial state $Z_{t_{0}}$, the requirements for rational expectations equilibrium (i.e., the structural equations (2.1), and a set of additional constraints of the form

$$
\left.\tilde{E} z_{t_{0}}=\bar{e} \equiv \tilde{E}\left[f_{0}+f_{Z} \bar{Z}_{t_{0}}+f_{s} \bar{s}_{t_{0}}\right)\right]
$$

on the initial behavior of the non-predetermined endogenous variables.

Here the additional constraints (3.2) restrict the possible values of the initial non-predetermined variables $z_{t_{0}}$ only insofar as expectations regarding the values of these variables should have affected equilibrium determination at earlier dates. Note also that these additional constraints refer only to period $t_{0}$ (the period in which the optimality of commitment to the rule is being considered). Thus the fact that it is judged desirable to commit to a rule that should imply evolution of the non-predetermined variables according to (3.1) in periods $t>t_{0}$ does not depend on any assumed constraint on the evolution in those periods, other than the constraint that the expected evolution represent a rational-expectations equilibrium. Hence in submitting to the constraints (3.2) in period $t_{0}$, the central bank is choosing to conform to a rule of conduct to which it should have wished to be expected to conform,

\footnotetext{
${ }^{16}$ We consider only the optimal plan among possible plans satisfying some uniform bound, since the Taylor-series approximations involved in the derivation of our quadratic loss function and linear equilibrium conditions are generally valid only locally. Unbounded paths $\left\{y_{t}\right\}$ that yield a lower value of the objective (1.2) need not correspond to any feasible equilibrium of the exact model, and so are not considered here. Of course, our interest in policies that are optimal in this local sense depends on a belief that the optimal equilibrium of the exact model is in fact one that involves only small departures from the steady state around which we have linearized the equilibrium conditions; in that case, the locally optimal policy defined here should represent a linear approximation to the true optimal policy.
} 
had the question been considered at an earlier date without any restriction of this kind upon date- $t_{0}$ conduct.

Because the additional constraints (3.2) refer only to outcomes in period $t_{0}$, the equilibrium dynamics resulting from commitment to a policy that is optimal from a timeless perspective involve the same responses to unanticipated shocks in all periods $t>t_{0}$ as would occur under the $t_{0}$-optimal plan, i.e., the evolution from date $t_{0}$ onward that would minimize (1.2) in the absence of the additional constraints. (Because of the assumed linearity of our equilibrium conditions (2.1), the planned linear response to an unanticipated shock at date $t$ has no effect on the constraints on possible outcomes at earlier dates. This implies that the optimal response to a shock at date $t>t_{0}$ is independent of the constraint values $\bar{e}$ imposed on the choices for date $t_{0}$, and indeed is the same even if the constraints (3.2) are omitted from the minimization problem.) Furthermore, if (as we shall assume below) the solution to the optimization problem stated in part (ii) of the above definition is one under which the expected long-run average values of the endogenous variables are independent of the initial conditions, then it follows that the long-run average values under a rule that is optimal from a timeless perspective are the same as those under commitment to the $t_{0}$-optimal plan.

Thus commitment to an optimal rule in this sense eliminates both the average inflation bias and the sub-optimal dynamic responses to shocks (sometimes called "stabilization bias") that are typically associated with discretionary policy. The equilibrium evolution from $t_{0}$ onward under the optimal policy from a timeless perspective differs from the $t_{0}$-optimal plan only in a transitory, deterministic component of the paths of the endogenous variables. Departure (in general) from the $t_{0}$-optimal choice of this component is necessary in order for policy to be time-consistent; and the central bank's willingness to depart from the $t_{0}$-optimal plan in this single respect can be understood as following from unwillingness to exploit previously existing expectations at the time that the policy commitment is chosen.

In (3.1), the non-predetermined variables are allowed to depend on vectors of predetermined and/or exogenous variables that may include more elements than the vectors of predetermined variables $Z_{t}$ and exogenous states $s_{t}$ that suffice to define the set of possible fu- 
ture evolutions of the economy (consistent with rational-expectations equilibrium) from date $t$ onward. Such dependence on additional state variables will occur (under the assumption of determinacy of equilibrium) only insofar as the policy rule itself involves those additional variables - that is, insofar as policy is not purely forward-looking. In general, we find that an optimal policy rule will not be purely forward-looking, for reasons that are closely related to the observation that in general the equilibrium evolution from date $t_{0}$ onward under the optimal rule will not minimize (1.2) if the constraints (3.2) are not imposed. The optimal plan (from the timeless perspective) involves conformity to constraints that insure that the central bank internalizes the effects of its predictable current policy upon prior expectations, and hence upon its ability to achieve its stabilization objectives at earlier dates. But this implies that policy will depend not solely upon the state variables that determine the economy's possible evolution from now on, but also upon past conditions as well, insofar as these would matter to a determination of the value in the past of having had the private sector expect a different sort of policy now. This requires an optimal policy rule to incorporate additional history-dependence. ${ }^{17}$

\section{Robustness to Alternative Types of Disturbances}

In the case of a particular detailed specification of a quantitative model of the economy including not just a specification of the coefficients of the various structural equations, but also a precise specification of the statistical properties of all of the exogenous shock processes - it is a standard exercise to evaluate alternative policy rules through stochastic simulation or other methods, and one may thus seek to find the optimal rule within some parametric family. ${ }^{18}$ In the case of a linear-quadratic policy problem of the kind that we assume here, it is even fairly straightforward to compute an optimal policy. However, the practical usefulness of the results of such analyses is often doubted, on the ground that the conclusions obtained obviously depend upon the precise model of the economy assumed, whereas an actual central

\footnotetext{
${ }^{17}$ See Woodford (2000; 2002, chap. 7) for additional discussion.

${ }^{18}$ See, e.g., Taylor (1999) for a collection of exercises of this kind in the context of variety of types of larger or smaller quantitative models of the monetary transmission mechanism.
} 
banker is unlikely ever to give assent to any such model as a complete description of what he or she believes about the structure of the economy.

In particular, the actual conduct of monetary policy typically involves detailed discussion of current conditions (and projections for the next year or two), taking account of a wide range of sources of information, both qualitative and quantitative. Central bankers are unlikely to be willing to restrict themselves to the consideration of only a small number of statistics when considering the economic outlook, and to be willing to identify the current situation with a particular realization from a probability distribution that could have been described in advance, and used in conducting the stochastic simulations used to analyze alternative policy rules. The fact is, there are always special circumstances of one sort or another, and it is difficult to imagine that central bankers will ever be willing to implement a rule that has been shown to be optimal only on the assumption that circumstances like those currently faced can never occur.

Yet much of the richness of actual central-bank discussions of the economic outlook can be allowed for within the context of a specific quantitative model of the economy, if we simply allow the additive disturbance terms in our equations to be extremely various in character. That is to say, rather than assuming a small number of stochastic disturbances (say, one per structural equation) with specified statistical properties (say, AR(1) processes with innovation variances and coefficients of serial correlation that have been estimated so as to match certain moments of historical time series), one should recognize that each of the model equations can be perturbed by any of vast number of types of disturbances, each with different statistical properties. Thus even if all of the relevant disturbances can be usefully characterized in terms of how much they shift each of the finite number of structural equations of one's economic model, they will differ in terms of how long it is expected to take for the shock to have its maximum effect, the rate at which this effect will thereafter decay, and so on; an infinite number of parameters will be required to precisely describe the effects of a given type of shock, even in the case of a very small economic model. One should accept that the number of distinct types of disturbances that may occur in practice is too 
large to imagine listing them all once and for all, let alone estimating all of the parameters of a complete description of their statistical properties and their relative likelihoods. At the same time, one should recognize that central bankers will have fairly specific ideas about the character of the particular disturbances that have most recently affected their national economies, and will accordingly require that an analysis of optimal policy be able to address the specific question of how one should optimally respond to those particular disturbances. ${ }^{19}$

Interestingly, one can base policy upon an analysis that allows for the possibility of the specific kinds of disturbances that happen most recently to have occurred, without policy reducing to discretionary optimization. This is because it is possible to choose a policy rule, commitment to which would be optimal (from the timeless perspective explained above) regardless of the statistical properties of the additive disturbances to the model equations, and indeed even if these can only be fully specified by a statistical model with millions of parameters. Specifically, we show below that it can be possible to establish that a particular linear rule with constant coefficients is optimal under the hypothesis that the disturbance terms have unconditional means of zero, without any further assumptions about their statistical properties.

A given structural disturbance $s_{j t}$ need only be assumed to be of the form

$$
s_{j t}=\sum_{k=0}^{\infty} \sum_{m} \alpha_{m, k}^{j} \epsilon_{m, t-k},
$$

where the index $m$ ranges over a possibly infinite list of possible types of disturbances, and where each of the random variables $\left\{\epsilon_{m, t}\right\}$ is an i.i.d. mean-zero variable, each with its own probability distribution. For each disturbance, the coefficients $\left\{\alpha_{m, k}^{j}\right\}$ indicate the degree to which it is forecastable in advance and the length of time for which its effects persist; this

\footnotetext{
${ }^{19}$ Our point is related, but not identical, to Svensson's (2001) advocacy of targeting rules on the ground that they allow the use of "judgment". Svensson assumes that central bankers have information about certain state variables, and could use this knowledge in implementing a targeting rule (by using the information in its forecasts of the target variables), but cannot adopt a rule that refers explicitly to those states. His analysis is thus based upon a distinction between disturbances to which policy may directly respond and others to which it must not. Our own emphasis is instead upon the advantage, from the point of view of simplicity, of commitment to a rule that does not require explicit reference to particular types of disturbances at all, and not simply avoiding mention of certain particularly difficult-to-describe disturbances.
} 
infinite list of parameters may be different for each disturbance. We may also suppose that for most if not all of the shocks, the distribution from which $\epsilon_{m, t}$ is drawn each period has a very large atom at the value zero, so that a shock of this type (of non-zero magnitude) is observed only infrequently. Thus it is logically possible both to assume that the central bank has a correct understanding of the nature of all of the disturbances that have hit the economy to date, and at the same time to suppose that it does not possess a complete list of all of the possible future disturbances, or sufficient data to estimate their likelihood of occurrence within a certain time interval. Under the assumption that the (innumerable) shocks are all mean-zero, such a bank would still be able to correctly evaluate both the history of the disturbance term, $s_{j, t-k}$ for all $k \geq 0$, and the conditional expectation of its future path, $\mathrm{E}_{t} s_{j, T}$ for all $T \geq t+1$. In a log-linear (certainty-equivalent) model of the kind that we use to approximate our account of the monetary transmission mechanism, assumption of a (symmetric-information) rational-expectations equilibrium requires that we assume that everyone in the economy shares a common (correct) evaluation of these conditional expectations, though there need not be correct knowledge of or even agreement upon other aspects of the probability distribution for the future disturbances; it is for this reason that we assume correct knowledge of the average values of the disturbance terms, which we may then without loss of generality assume to be zero.

We seek a policy rule with the following property.

Definition. A time-invariant policy rule (1.1) is robustly optimal if it is optimal from a timeless perspective, regardless of the specification of the coefficients $\left\{\alpha_{m, k}^{j}\right\}$ in $(4.1)$, and regardless of the distributions from which the innovations $\epsilon_{m, t}$ are drawn (except that these must be bounded and have mean zero).

The key to the possibility of a robustly optimal policy rule in this sense is the certaintyequivalence of optimal policy in the case of a linear-quadratic optimal policy problem of the kind that we consider here. This means that the central bank's optimal instrument setting 
may depend upon the conditional expectations of disturbances at various future horizons, as well as upon current and past disturbance terms, but that it is independent of other details of the probability distribution of future disturbances, and thus can be expressed without any need to enumerate or assign probabilities to all of the possible types of future disturbances. In fact, as we shall see, it is possible to express optimal policy in terms of an instrument rule or targeting rule that makes no reference even to the disturbances that have already occurred, except insofar as these affect the bank's estimates of (and projections of the future paths of) its target variables (such as the output gap).

The quest for policy rules that are robustly optimal allows us to obtain substantially sharper conclusions about the nature of an optimal rule. Indeed, it is only under this additional stipulation that questions about the optimal form of interest-rate rule become meaningful. ${ }^{20}$ For example, the question of the degree to which it is optimal for central bank policy to be based on forecasts has no interesting answer if an "optimal policy" is required only to support the optimal equilibrium in the case of a particular specification of the statistical properties of the disturbance processes. For if a forecast-based policy rule can be found that is consistent with the desired equilibrium, one can necessarily also obtain a purely backward-looking rule (an explicit instrument rule in the sense defined above) by substituting for the forecast the particular function of predetermined and exogenous variables that represents the rational forecast in the case of the particular disturbance processes and the particular equilibrium. However, the mapping between these two alternative representations of policy is not invariant under a change in the assumed statistical properties of the disturbances. Hence the two representations need not be equally robust to alternative specifications of the disturbance processes, and it becomes possible to ask which form of rule is robustly optimal. To anticipate our conclusions, we shall find that robustly optimal

\footnotetext{
${ }^{20}$ Here our perspective differs from much previous literature, where, for example, the question of the optimal forecast horizon is addressed by computing the equilibria associated with particular tightly parameterized families of forecast-based policy rules with different forecast horizons (e.g., Black et al., 1997; Batini and Haldane, 1999; Rudebusch and Svensson, 1999; Levin et al., 2001; Batini and Pearlman, 2002). The results obtained in these studies are highly dependent upon the particular restrictive forms of policy rules assumed, that are arbitrarily chosen, though appealing in their simplicity. Our primary interest here is instead in what can be achieved by various classes of relatively flexibly specified classes of policy rules.
} 
rules are possible, but that they typically cannot be explicit instrument rules. Instead, rules with this property involve projections at least of current endogenous variables, and generally projections at least some distance into the future as well.

The particular form of robustness just discussed does not, of course, represent the only practically relevant kind of uncertainty that will exist about the validity of a given model of the monetary transmission mechanism. In fact there will also be considerable uncertainty about the correct specification of the non-stochastic terms in the model equations as well, and a policy rule that is shown to be optimal only under the assumption that all structural coefficients are known with certainty will still have to face questions about how far from optimal it might be under alternative assumptions. We do not here consider how a concern for other sorts of "robustness" should affect our choice of a policy rule. ${ }^{21}$ This is a much harder problem, since it is not generally possible to find a single rule that will be optimal regardless of which of a range of possible model specifications are correct. Instead, one faces a tension between the goals of minimizing expected losses according to the model that represents one's best guess, and of choosing a rule that will also not be too bad according to alternative models; as a result, the question of how much one should be concerned about the possibility of various alternative possibilities arises. We here note only that this important issue is not too closely related to the kind of robustness treated here.

Even with the further stipulation of robust optimality, there will be many possible policy rules with the desired property. Hence we further narrow our search to the category of direct rules - rules that involve only the target variables $\tau_{t}$, though they may involve both leads and lags of these ${ }^{22}$ - for the reasons discussed in section 1 . We show in section 6 that it is quite generally possible to find a time-invariant direct policy rule that is robustly optimal. Indeed, even under these requirements, we cannot generally isolate a single uniquely optimal

\footnotetext{
${ }^{21}$ See, however, Hansen and Sargent (2000) and Giannoni (2001, 2002) for analyses of monetary policy intended to be robust against other types of model uncertainty, in the context of forward-looking models like those considered here.

${ }^{22}$ In fact, the optimal rules that we display involve only the target variables, whether or not the instrument happens to be a target variable (though in general we shall assume that it is). Thus our optimal rules can always be interpreted as targeting rules, which is the interpretation preferred in Svensson (2001).
} 
rule, though our attention is narrowed to a small number of possibilities.

\section{Characterizing Optimal Equilibrium Dynamics}

Here we begin our exposition of a general approach to the construction of robustly optimal direct policy rules, in the case of a linear-quadratic policy problem of the kind described above. We begin by characterizing the equilibrium dynamics that would be judged to be optimal from a timeless perspective; we then turn, in the next section, to the construction of a policy rule that implies a determinate equilibrium of this kind.

We begin by characterizing the state-contingent path for the endogenous variables (including the instrument) from some date $t_{0}$ onward that minimizes (1.2) subject to the constraints that the evolution satisfy equations (2.1) at each date $t \geq t_{0}$, given an initial value $Z_{t_{0}}$ for the predetermined variables, and subject to the additional constraints (3.2). As in Woodford (1999a), the Lagrangian for this minimization problem can be written in the form

$$
\mathcal{L}_{t_{0}}=\mathrm{E}_{t_{0}}\left\{\sum_{t=t_{0}}^{\infty} \beta^{t-t_{0}}\left[L\left(y_{t}\right)+\varphi_{t+1}^{\prime} \tilde{A} y_{t}-\beta^{-1} \varphi_{t}^{\prime} \tilde{I} y_{t}\right]\right\},
$$

where

$$
\tilde{A} \equiv\left[\begin{array}{ll}
A & B
\end{array}\right], \quad \tilde{I} \equiv\left[\begin{array}{ll}
\hat{I} & 0
\end{array}\right]
$$

Here $L\left(y_{t}\right)$ is the period loss $L_{t}$ expressed as a quadratic function of $y_{t}$, and $\varphi_{t+1}$ is the vector of Lagrange multipliers associated with the constraints (2.1).

The conditional expectation has been eliminated from the term $\mathrm{E}_{t} z_{t+1}$ in these constraints, using the law of iterated expectations (since the entire expression is conditional upon information at date $t_{0}$ in any event). However, because the last $n_{z}$ constraints hold only in conditional expectation, the last $n_{z}$ elements of $\varphi_{t+1}$ must be measurable with respect to period $t$ information (i.e., cannot depend upon shocks at date $t+1$ ). We therefore introduce the notation

$$
\varphi_{t+1} \equiv\left[\begin{array}{c}
\xi_{t+1} \\
\Xi_{t}
\end{array}\right]
$$


where the partition is conformable to the partition of the rows of (2.1); the different time subscript on $\Xi_{t}$ (as in Svensson and Woodford, 2000, 2001) serves as a reminder that these elements of the vector are determined a period earlier. We have suppressed the terms in the Lagrangian involving the exogenous disturbances $s_{t}$, as these do not matter for the first-order conditions derived below. Finally, the term

$$
\varphi_{t_{0}}^{\prime} \tilde{I} y_{t_{0}}=\xi_{t_{0}}^{\prime} Z_{t_{0}}+\Xi_{t_{0}-1}^{\prime} \tilde{E} z_{t_{0}}
$$

has been added to the Lagrangian in (5.1). The first term on the right-hand side represents the constraints imposed by the given initial values $Z_{t_{0}}$ for the predetermined variables, while the second term represents the constraints (3.2).

The same Lagrangian can be used to characterize the $t_{0}$-optimal plan, i.e., the timeinconsistent "optimal commitment" that is often considered in the literature on rules versus discretion. This is the path for the evolution of the endogenous variables from $t_{0}$ onward that minimizes the same criterion, in the absence of any constraints of the form (3.2). The Lagrangian for such a minimization problem is of the same form as (5.1), except that the final term $\Xi_{t_{0}-1}^{\prime} \tilde{E} z_{t_{0}}$ should be omitted. Alternatively, we may write the Lagrangian in the form (5.1) for the sake of symmetry, but impose the stipulation that

$$
\Xi_{t_{0}-1}=0
$$

Thus the $t_{0}$-optimal plan also minimizes a Lagrangian of the form (5.1), but with (5.2) imposed as an additional initial condition, rather than (3.2).

Differentiating the Lagrangian (5.1) with respect to the endogenous variables $y_{t}$, we obtain the first-order conditions

$$
\tilde{A}^{\prime} \mathrm{E}_{t} \varphi_{t+1}+T^{\prime} W\left(\tau_{t}-\tau^{*}\right)-\beta^{-1} \tilde{I}^{\prime} \varphi_{t}=0
$$

for each $t \geq t_{0}$. An optimal plan must also satisfy a transversality condition, but this is necessarily satisfied in the case of any bounded solution to the structural equations, and we have already noted that we shall restrict our attention to bounded solutions. Thus any 
bounded processes for the endogenous variables $\left\{y_{t}\right\}$ and the Lagrange multipliers $\left\{\varphi_{t}\right\}$ for dates $t \geq t_{0}$, that are consistent with the initial conditions for $Z_{t_{0}}$ and with (5.2), and that satisfy the structural equations (2.1) and the first-order conditions (5.3) for each $t \geq t_{0}$, describe a $t_{0}$-optimal commitment. Alternatively, any bounded processes consistent with the initial conditions $Z_{t_{0}}$ and (3.2), and satisfying (2.1) and (5.3) for each $t \geq t_{0}$, satisfy part (ii) of the requirements stated in section 3 for optimality from a timeless perspective.

In what follows we shall maintain the following assumption about our linear-quadratic policy problem.

Assumption 1. In the case of any bounded disturbance processes and any initial conditions $Z_{t_{0}}$ and $\Xi_{t_{0}-1}$, there is a unique bounded solution to the system of equations consisting of (2.1) and (5.3). In particular, in the case of any such disturbance process and any initial conditions $Z_{t_{0}}$, there is a unique bounded solution to the system of equations consisting of (2.1), (5.2) and (5.3). Thus there exists a unique bounded $t_{0}$-optimal plan $\left\{y_{t}\right\}$, to which there is associated a bounded process $\left\{\varphi_{t}\right\}$ for the Lagrange multipliers as well. ${ }^{23}$

In fact, under quite weak assumptions a linear-quadratic problem of this kind has a unique optimum (subject to bounds on the rate at which the endogenous variables may grow asymptotically), in which $\beta^{t / 2} y_{t}$ and $\beta^{t / 2} \varphi_{t}$ are bounded. Assumption 1 thus represents only a small strengthening of the canonical assumptions, so that the optimal processes $\left\{y_{t}\right\}$ and $\left\{\varphi_{t}\right\}$ are bounded without the rescaling. ${ }^{24}$ It should also be noted that under the canonical assumptions, if there exists a bounded optimal plan — as we must assume, in most applications of interest, in order to justify working with a log-linear approximation to the exact equilibrium conditions in our characterization of optimal policy - then the first order conditions must have a unique bounded solution, as assumed here, for other bounded solutions would have

\footnotetext{
${ }^{23}$ By a bounded optimal plan we mean a process $\left\{y_{t}\right\}$ that minimizes (1.2) among the class of bounded processes, subject to the other stated constraints.

${ }^{24}$ The asymptotic growth rates of these variables depend upon certain roots of a characteristic polynomial, that are necessarily no larger than $\beta^{-1 / 2}$, but will for most parameter values be smaller, and can easily be smaller than one. If $\beta$ is only slightly less than one, Assumption 1 will hold for a large range of parameter values.
} 
to correspond to alternative optimal plans.

Assumption 1 also states that there is a unique bounded solution regardless of the assumed value for $\Xi_{t_{0}-1}$, and not only in the case of the value $\Xi_{t_{0}-1}$ associated with the $t_{0}$-optimal commitment. But in the generic case, the existence of a unique bounded solution to the system consisting of (2.1) and (5.3) depends only on the roots of a characteristic polynomial associated with the coefficients of these equations, and is independent of the assumed vectors of initial conditions $Z_{t_{0}}$ and $\Xi_{t_{0}-1}$. Hence a unique bounded solution will almost always exist for arbitrary $Z_{t_{0}}$ and $\Xi_{t_{0}-1}$ if there exists a unique bounded $t_{0}$-optimal plan for arbitrary $Z_{t_{0}}$.

It follows from the definition in section 3 that in the equilibrium associated with a policy rule that is optimal from a timeless perspective, the evolution of the endogenous variables from date $t_{0}$ onward must satisfy (2.1) and (5.3) for each $t \geq t_{0}$, for some choice of the initial multipliers $\Xi_{t_{0}-1}$. Under Assumption 1, there is a unique bounded solution corresponding to any given specification of $\Xi_{t_{0}-1}$, and in particular a unique implied value for

$$
\tilde{E} z_{t_{0}}=e_{0}+e_{Z} Z_{t_{0}}+e_{s} s_{t_{0}}+e_{\Xi} \Xi_{t_{0}-1} .
$$

Hence corresponding to any specification of $\Xi_{t_{0}-1}$ there is an associated value of $\bar{e}$, given by the right-hand side of (5.4), such that the unique bounded solution corresponding to this choice of $\Xi_{t_{0}-1}$ represents the optimal evolution from date $t_{0}$ onward subject to the additional constraints (3.2).

Our definition of optimality from a timeless perspective can then be equivalently restated as follows. A policy rule that determines a unique bounded equilibrium is optimal from a timeless perspective if

(i) the equilibrium satisfies a time-invariant relation of the form (3.1);

(ii) in this equilibrium, the endogenous variables $y_{t}$ for $t \geq t_{0}$ evolve according to the unique bounded solution to (2.1) and (5.3) for $t \geq t_{0}$, consistent with the given initial conditions $Z_{t_{0}}$ and some initial Lagrange multipliers $\Xi_{t_{0}-1}$; and 
(iii) the initial multipliers are given by a linear rule of the form

$$
\Xi_{t_{0}-1}=g_{0}+g_{Z} \bar{Z}_{t_{0}}+g_{s} \bar{s}_{t_{0}}
$$

where the coefficients $g_{0}, g_{Z}, g_{s}$ are such that in the equilibrium,

$$
\tilde{E} z_{t}=e_{0}+e_{Z} Z_{t}+e_{s} s_{t}+e_{\Xi}\left[g_{0}+g_{Z} \bar{Z}_{t}+g_{s} \bar{s}_{t}\right]
$$

at all dates $t \geq t_{0}$ and under all possible realizations of the exogenous disturbances; that is, the right-hand side of (3.1) pre-multiplied by $\tilde{E}$ coincides with the right-hand side of (5.6).

Here condition (ii) guarantees that the evolution of the endogenous variables from $t_{0}$ onward is optimal subject to some constraint of the form (3.2), while condition (iii) ensures that the constraint value $\bar{e}$ for which this is true is derivable from the initial conditions $\bar{Z}_{t_{0}}, \bar{s}_{t_{0}}$ using a time-invariant rule (5.6) that one wishes to commit to satisfying in all subsequent periods.

One case in which we may be sure that the rule (5.5) satisfies (5.6), of course, is if the corresponding relation

$$
\Xi_{t-1}=g_{0}+g_{Z} \bar{Z}_{t}+g_{s} \bar{s}_{t}
$$

holds for all $t>t_{0}$ in the unique bounded solution to the system consisting of (2.1) and (5.3) consistent with initial multipliers (5.5). This is the approach that we shall take to the construction of a policy rule that is optimal from a timeless perspective. Note that in equilibrium, the multipliers $\Xi_{t-1}$ for any $t>t_{0}$ depend only on the economy's state at date $t-1$ (hence our choice of subscript). Thus (5.7) must have this property; that is, $g_{Z}$ and $g_{s}$ must put non-zero weight only on elements of $\bar{Z}_{t}$ and $\bar{s}_{t}$ that are known at date $t-1$.

Our proposed approach can now be sketched as follows. First, our structural equations (2.1) and stabilization objectives (1.2) allow us to define the optimal dynamics corresponding to any specification $\Xi_{t_{0}-1}$ of the initial Lagrange multipliers. We then seek to find a rule (5.5) for choosing the initial multipliers with the property that the implied equilibrium satisfies the corresponding relation (5.7) at all later dates as well. This then determines the particular equilibrium evolution of the economy that we wish to implement. Finally, 
we seek to formulate a time-invariant target criterion referring only to the projected path of the target variables, with the property that commitment to fulfill this target criterion in all periods $t \geq t_{0}$ implies a determinate rational-expectations equilibrium in which the endogenous variables evolve in the desired way.

\section{Existence of Optimal Policy Rules}

We now turn to the question of finding a policy rule that can be expected to bring about equilibrium dynamics of the kind characterized in the previous section. The key insight is that one can design a time-invariant target criterion (1.1) that implies that the endogenous variables must evolve in a way that is consistent with the system of first-order conditions (5.3) that characterize an optimal commitment, ${ }^{25}$ under a particular rule (5.5) for choice of the initial Lagrange multipliers. This kind of rule necessarily implies a determinate equilibrium, because the system of first-order conditions has (under Assumption 1) a unique bounded solution. And while the equilibrium will in general not coincide in its transition dynamics with the $t_{0}$-optimal commitment - for the initial Lagrange multipliers must generally not be set equal to zero, in order for the proposed rule to be time-consistent — the economy's equilibrium evolution is optimal from the timeless perspective defined in section 3. Moreover, because the first-order conditions involve only the target variables (in addition to the Lagrange multipliers, that we eliminate in order to derive the policy rule), the optimal rule is a direct rule. And because the first-order conditions are independent of the assumed statistical properties of the disturbance processes, the optimal rule is robust to changes in that aspect of the model specification.

In order to apply the method presented here, we require a further assumption about the matrices $\hat{I}$ and $A$ in the structural equations (2.1).

Assumption 2. The characteristic polynomial (2.3) has at least $n_{Z}$ roots such that

\footnotetext{
${ }^{25}$ Our approach generalizes the derivation of optimal "specific targeting rules" in Svensson (1997), Svensson and Woodford (1999), and Svensson (2001).
} 
$\left|\mu_{i}\right|<\beta^{-1}$, i.e., at least $\operatorname{rank}(A)-n_{z}$ non-zero roots satisfying that bound, in addition to the zero root that is repeated $n-\operatorname{rank}(A)$ times. Furthermore, it is possible to select a set of exactly $n_{Z}$ roots, including the $n-\operatorname{rank}(A)$ zeroes, such that either (i) the roots in this set consist entirely of real roots and of complex pairs, both elements of which belong to the set; or (ii) there is a single complex root $\mu$ in the set, the complex conjugate of which is not also in the set, and this root is such that $\left|\operatorname{Re} \mu^{-1}\right|>\beta$.

In the generic case, the first part of Assumption 2 implies that in the case of a constant instrument setting, $i_{t}=\bar{\imath}$ for all $t$, there exists one or more solutions to the structural equations (2.1) in which $\beta^{t} y_{t}$ remains bounded for all $t$, regardless of the initial conditions $Z_{t_{0}}$ and regardless of the specification of the disturbance processes, as long as the disturbances are themselves bounded. This means that policy need not be adjusted in any special way in order to prevent explosive (at least, unduly explosive) dynamics; a non-explosive equilibrium path for the endogenous variables will exist as long as explosive dynamics are not required by the violent adjustment of the policy instrument. We note furthermore that this part of Assumption 2 is implied by the Sargent-Wallace property.

The further stipulation regarding the set of exactly $n_{Z}$ roots is a relatively weak additional restriction. It is necessarily possible to choose a set of roots with property (i), except in the case that the non-zero roots satisfying $\left|\mu_{i}\right|<\beta^{-1}$ are all complex, and $\operatorname{rank}(A)-n_{z}$ is odd. Even in this case, it is necessarily possible to choose a set of $n_{Z}$ roots in which there is only one complex root such that the complex conjugate is not also in the set. Thus the only real restriction is the further stipulation that this root be such that $\left|\operatorname{Re} \mu^{-1}\right|>\beta$, which is stronger than the restriction already assumed on the modulus of the root.

We shall also assume that these matrices satisfy two additional technical assumptions, Assumptions 3 and 4, stated in the Appendix. Here we simply note that both of the latter two assumptions hold for generic matrices $A$ and $\tilde{E}$, of $\operatorname{arbitrary} \operatorname{ranks} 0 \leq \operatorname{rank}(\tilde{E}) \leq n_{z}$ and $n_{z} \leq \operatorname{rank}(A) \leq n$.

We now recall that conditions (5.3) may be separated into two sets of first-order condi- 
tions,

$$
\begin{gathered}
A^{\prime} \mathrm{E}_{t} \varphi_{t+1}+\tilde{T}^{\prime} W\left(\tau_{t}-\tau^{*}\right)-\beta^{-1} \hat{I}^{\prime} \varphi_{t}=0 \\
B_{2}^{\prime} \Xi_{t}+T^{i \prime} W\left(\tau_{t}-\tau^{*}\right)=0
\end{gathered}
$$

where the columns of

$$
T \equiv\left[\begin{array}{ll}
\tilde{T} & T^{i}
\end{array}\right]
$$

are partitioned conformably with those of $\tilde{A}$. Given any bounded process for the evolution of the target variables, it is possible to find a process for the multipliers that satisfies conditions (6.1).

Proposition 1. Under Assumptions $2-4$, there exist (real-valued) matrices $\Lambda$ and $\Upsilon$ and linear operators $Q\left(L^{-1}\right)$ and $R\left(L^{-1}\right)$ such that bounded processes $\left\{\varphi_{t}\right\}$ and $\left\{\tau_{t}\right\}$ satisfy (6.1) for all $t \geq t_{0}$ if and only if they satisfy the conditions

$$
\begin{aligned}
\Xi_{t} & =\Lambda \Xi_{t-1}+\mathrm{E}_{t}\left[Q\left(L^{-1}\right) \tilde{T}^{\prime} W\left(\tau_{t}-\tau^{*}\right)\right] \\
\xi_{t} & =\Upsilon \Xi_{t-1}+\mathrm{E}_{t}\left[R\left(L^{-1}\right) \tilde{T}^{\prime} W\left(\tau_{t}-\tau^{*}\right)\right]
\end{aligned}
$$

for all $t \geq t_{0}$. These linear operators are such that $Q\left(L^{-1}\right) x_{t}$ and $R\left(L^{-1}\right) x_{t}$ are well-defined and bounded processes in the case of any bounded process $x_{t}$.

The proof is given in the Appendix, along with the statement of Assumptions 3 and 4. In the case that there are exactly $n_{Z}$ roots of $(2.3)$ such that $\left|\mu_{i}\right|<\beta^{-1}, \Lambda, \Upsilon, Q$ and $R$ are unique, and there is a unique solution to (6.1) for the evolution of the multipliers $\left\{\varphi_{t}\right\}$ given a process the evolution of the target variables $\left\{\tau_{t}\right\}$ and an initial condition $\Xi_{t_{0}-1}$. If instead there are more than $n_{Z}$ such roots, there are many possible solutions for the evolution of the multipliers. Nonetheless, it is possible to select a solution in which $\Xi_{t}$ and $\xi_{t}$ are timeinvariant functions of the lagged value $\Xi_{t-1}$ and the expected path of the target variables from date $t$ onward, as indicated in (6.3) - (6.4). In this case the values of $\Lambda, \Upsilon, Q$ and $R$ are not uniquely determined, but there are only a small number of possibilities that work, 
corresponding to the different ways in which it is possible to select a set of $n_{Z}$ roots of (2.3) with modulus $\left|\mu_{i}\right|<\beta^{-1}$.

We next observe that for any square matrix $\Lambda$ of dimension $n_{z}$, there exist an $n_{z} \times n_{z}$ matrix polynomial $B(L)$ and a scalar polynomial $\alpha(L)$ such that

$$
B(L)(I-\Lambda L)=\alpha(L) I .
$$

Here $\alpha(L)=\operatorname{det}(I-\Lambda L)$, and $B(L)$ is the adjoint of $(I-\Lambda L)$, i.e., the transpose of the matrix of cofactors. Note that $\alpha(L)$ is of order $\operatorname{rank}(\Lambda)=\operatorname{rank}(\tilde{E}) \equiv k$, while $B(L)$ is of order $d \equiv \min \left(k, n_{z}-1\right)$. Premultiplying (6.3) by $B(L)$, we obtain

$$
\alpha(L) \Xi_{t}=B(L) \mathrm{E}_{t}\left[Q\left(L^{-1}\right) \tilde{T}^{\prime} W\left(\tau_{t}-\tau^{*}\right)\right]
$$

This equation holds for all $t \geq t_{0}+k$ if (6.3) holds for all $t \geq t_{0}$, and conversely, if (6.3) holds for all $t_{0} \leq t<t_{0}+k$ and the above condition holds for all $t \geq t_{0}+k$, it follows that (6.3) also holds for all $t \geq t_{0}+k$.

Finally, premultiplying this last equation by $B_{2}^{\prime}$ and using (6.2) to eliminate the $B_{2}^{\prime} \Xi_{t}$ terms, we obtain the condition

$$
\alpha(L) T^{i \prime} W\left(\tau_{t}-\tau^{*}\right)+B_{2}^{\prime} B(L) \mathrm{E}_{t}\left[Q\left(L^{-1}\right) \tilde{T}^{\prime} W\left(\tau_{t}-\tau^{*}\right)\right]=0,
$$

which involves only the target variables. Under the optimal once-and-for-all commitment chosen at date $t_{0}$, the evolution of the target variables will satisfy this criterion at all dates $t \geq t_{0}+k .^{26}$ Thus a commitment by the policymaker to enforce condition (6.6) each period is an example of a policy that can be justified on the ground that it would have been optimal to commit to such a policy in the case of any optimal commitment chosen at a date sufficiently far in the past.

Condition (6.6) is an example of a direct policy rule that takes the time-invariant form (1.1). Both are desirable properties of a policy rule, as discussed above. In order to express

\footnotetext{
${ }^{26}$ Under this optimal commitment, both (6.2) and (6.3) hold for all $t \geq t_{0}$. Once both (6.2) and (6.3) have held for the previous $k$ periods, it is possible to use the derivation in the text to show that (6.6) must hold.
} 
(6.6) in the form (1.1), it suffices that the vector $\bar{Z}_{t}$ be defined as

$$
\bar{Z}_{t} \equiv\left(Z_{t}^{\prime} q_{t-1}^{\prime} \ldots q_{t-d}^{\prime} v_{t-1} \ldots v_{t-k}\right)^{\prime}
$$

where

$$
\begin{gathered}
q_{t} \equiv \mathrm{E}_{t}\left[Q\left(L^{-1}\right) \tilde{T}^{\prime} W\left(\tau_{t}-\tau^{*}\right)\right] \\
v_{t} \equiv T^{i \prime} W\left(\tau_{t}-\tau^{*}\right)
\end{gathered}
$$

and that the vector $\bar{z}_{t}$ include not only the non-predetermined endogenous variables that matter for the current targets $\tau_{t}$, but also the conditional expectations of future target variables that are involved in $q_{t}$. In general, (6.7) will require that the vector $\bar{Z}_{t}$ include elements beyond those needed in order to specify the possible evolution of the target variables from period $t$ onward. ${ }^{27}$ Thus the rule is in general not purely forward-looking in the sense of Woodford (2000).

One can show quite generally that such a rule implies a determinate equilibrium. The following result relies upon an additional Assumption 5, stated in the Appendix; this condition is again one that holds for generic matrices of arbitrary rank.

Proposition 2. Under Assumptions 1 - 5, a commitment to ensure that condition (6.6) holds at all dates $t \geq t_{0}$ results in a determinate REE. In this equilibrium, $z_{t}$ each period is given by a linear relation of the form (3.1), where $\bar{s}_{t}=s_{t}$ (the exogenous state vector required to define the possible evolution of the target variables from date $t$ onward) and $\bar{Z}_{t}$ is given by $(6.7)$.

Furthermore, the bounded process $\left\{y_{t}\right\}$ associated with this equilibrium minimizes (1.2) among all possible bounded evolutions of the endogenous variables from date $t_{0}$ onward that are consistent with conditions (2.1), the initial conditions $Z_{t_{0}}$, and the additional constraints

$$
\tilde{E} z_{t_{0}}=\bar{e}
$$

\footnotetext{
${ }^{27}$ This is almost inevitably the case if $\Lambda \neq 0$, which is the case as long as $\tilde{E} \neq 0$, i.e., as long as the structural equations are at all forward-looking. See section A.1 of the Appendix for the relation between the rank of $\Lambda$ and the rank of $\tilde{E}$.
} 
where $\bar{e}$ is the function of $\bar{Z}_{t_{0}}$ and $s_{t_{0}}$ specified in (3.2).

The proof is given in the Appendix. The essential idea is that one can uniquely determine a vector of initial Lagrange multipliers $\Xi_{t_{0}-1}$ implied by the given initial conditions $\bar{Z}_{t_{0}}$, with the property that a bounded solution to the system consisting of (2.1) and (6.6) for all $t \geq t_{0}$ consistent with the initial conditions $\bar{Z}_{t_{0}}, s_{t_{0}}$ must correspond to a bounded solution to the system consisting of (2.1) and (5.3) consistent with the initial conditions $Z_{t_{0}}, \Xi_{t_{0}-1}, s_{t_{0}}$. The existence and uniqueness of the latter solution (under Assumption 1) then implies determinacy of rational expectations equilibrium under the commitment to the target criterion (6.6). In addition, the fact that the determinate equilibrium satisfies the system of equations consisting of (2.1) and (5.3) for some choice of the initial multipliers $\Xi_{t_{0}-1}$ implies that it is optimal subject to additional constraints of the form (6.10). The fact that the initial Lagrange multipliers are a time-invariant function of the initial conditions of the form (5.7) then guarantees that the additional constraints are the ones specified in (3.2).

This second part of Proposition 2 implies that policy rule (6.6) is optimal from a timeless perspective, in the sense defined in section 3. We note furthermore that the coefficients of this rule depend only upon the coefficients of the first-order conditions (5.3), which are independent of any assumptions about the statistical properties of the disturbances. Hence this policy rule is also robustly optimal, in the sense defined in section 4 .

\section{On the Form of Optimal Policy Rules}

The coefficient multiplying the current instrument setting $i_{t}$ in (6.6) is ${ }^{28}$

$$
T^{i \prime} W T^{i}+B_{2}^{\prime} Q(0) \tilde{T}^{\prime} W T^{i}
$$

If this quantity is non-zero, the rule can be interpreted as an implicit instrument rule. A case of particular interest is the following one.

\footnotetext{
${ }^{28}$ Note that the definitions above imply that $\alpha(0)=1, B(0)=I$.
} 
Assumption 6. The target variables can be partitioned as

$$
\tau_{t}=\left[\begin{array}{c}
\hat{\tau}_{t} \\
i_{t}
\end{array}\right],
$$

where $\hat{\tau}_{t}=\hat{T} \tilde{y}_{t}$ is a function only of $\tilde{y}_{t}$, the vector of endogenous variables other than the instrument, and the target values $\tau^{*}$ can similarly be partitioned into target values $\hat{\tau}^{*}$ and $i^{*}$. The matrix $W$ in (1.3) is also block-diagonal, so that

$$
L_{t}=\frac{1}{2}\left(\hat{\tau}_{t}-\hat{\tau}^{*}\right)^{\prime} \hat{W}\left(\hat{\tau}_{t}-\hat{\tau}^{*}\right)+\frac{\lambda_{i}}{2}\left(i_{t}-i^{*}\right)^{2},
$$

where $\hat{W}$ is a symmetric, positive-definite matrix, and $\lambda_{i} \geq 0$.

This assumption is satisfied in the examples considered in Giannoni and Woodford (2002). Under this assumption, the coefficient multiplying $i_{t}$ in (6.6) is simply $\lambda_{i}$. Then if $\lambda_{i}>0$ (i.e., if the policymaker's objective includes a concern for stabilization of the instrument), the optimal rule is an implicit instrument rule, that may be expressed in the form

$$
i_{t}=(1-\gamma(1)) i^{*}+\gamma(L) i_{t-1}-\lambda_{i}^{-1} B_{2}^{\prime} B(L) \mathrm{E}_{t}\left[Q\left(L^{-1}\right) \hat{T}^{\prime} \hat{W}\left(\hat{\tau}_{t}-\hat{\tau}^{*}\right)\right] .
$$

Here we have expressed the lag polynomial $\alpha(L)$ as $1-\gamma(L) L$, where $\gamma(L)$ is a polynomial of order $k-1$.

On the other hand, if $\lambda_{i}=0$, the optimal rule reduces to

$$
B_{2}^{\prime} B(L) \mathrm{E}_{t}\left[Q\left(L^{-1}\right) \hat{T}^{\prime} \hat{W}\left(\hat{\tau}_{t}-\hat{\tau}^{*}\right)\right]=0 .
$$

This is now a target criterion that does not involve the path of the instrument; it can nonetheless be regarded as specifying a well-defined policy rule, a pure "targeting rule". ${ }^{29}$ Because we have shown that there is a determinate rational-expectations equilibrium in which this criterion is fulfilled at all dates, the use of the instrument to ensure that this criterion holds is both feasible and suffices to uniquely determine an implied instrument setting at each date.

\footnotetext{
${ }^{29}$ As noted earlier, rule (6.6) can also be regarded as a targeting rule even in the case that the target criterion involves $i_{t}$. Svensson (2001) discusses optimal targeting rules of this form.
} 
We have shown under relatively general conditions that a robustly optimal direct policy rule will exist. But it does not follow that there is a single uniquely optimal rule in this sense. First of all, we have noted above that the $\Lambda, \Upsilon, Q$ and $R$ referred to in Proposition 1 may not be unique, so that (6.6) may not correspond to a unique specification of the policy rule. One way of obtaining a unique proposal of this kind, that we favor, is to select the representation of optimal policy of this kind that results in the lowest possible upper bound on the moduli of the eigenvalues of $\Lambda .^{30}$ This makes the dependence of the rule (6.6) upon lagged variables (and lagged expectations) as little as possible, so we call this the minimally history-dependent rule. As is discussed further in the Appendix (and illustrated in the analysis of the model with inflation inertia in Giannoni and Woodford, 2002), this choice also reduces unnecessary dependence upon forecasts of target variables far in the future in the statement of the rule.

But this does not suffice to resolve the question of uniqueness, for a rule need not be of the form (6.6) at all to be a robustly optimal direct rule. Any rule of the form

$$
a(L)^{\prime} \mathrm{E}_{t}\left[\Psi\left(L^{-1}\right)\left(\tau_{t}-\tau^{*}\right)\right]=0
$$

is equivalent to the rule

$$
\mathrm{E}_{t}\left\{\phi\left(L^{-1}\right) a(L)^{\prime} \mathrm{E}_{t}\left[\Psi\left(L^{-1}\right)\left(\tau_{t}-\tau^{*}\right)\right]\right\}=0,
$$

where $\phi\left(L^{-1}\right)$ is any invertible (scalar) polynomial function of the inverse lag operator, i.e., any polynomial such that all roots of $\phi(\mu)=0$ are outside the unit circle. These rules are equivalent in the sense that a bounded process $\left\{\tau_{t}\right\}$ satisfies (7.3) if and only if it satisfies (7.4). One might suppose that the form (7.4) is necessarily a more complex specification, on the ground that it involves projections of the target variables farther in the future, and therefore that (7.3) would unambiguously be preferred. But this need not be so. If one or more of the lag polynomials $a_{i}(L)$ can be factored as

$$
a_{i}(L)=\gamma_{1}(L) \gamma_{2}(L)
$$

\footnotetext{
${ }^{30}$ The construction of this particular rule of the form (6.6) is discussed in section A.2.3 of the Appendix.
} 
where $\gamma_{1}(L)$ is a lag polynomial of order $m \geq 1$ such that all roots of $\gamma_{1}(\mu)=0$ are inside the unit circle, we may choose $\phi\left(L^{-1}\right)=\tilde{\gamma}_{1}\left(L^{-1}\right)^{-1}$, where $\tilde{\gamma}_{1}\left(L^{-1}\right) \equiv \gamma_{1}(L) L^{-m}$ is a polynomial of order $m$ with all of its zeroes outside the unit circle. In this case,

$$
\mathrm{E}_{t}\left\{\phi\left(L^{-1}\right) a_{i}(L) x_{t}\right\}=\gamma_{2}(L) x_{t-m}
$$

for any bounded variable $x_{t}$, and this is a simpler function of $x_{t}$ (involving fewer lags and no more leads) than is $a_{i}(L) x_{t}$.

In particular, this possibility arises in the following case.

Assumption 7. The characteristic polynomial (2.3) has $n_{Z}+m$ roots such that $|\mu|<$ $\beta^{-1}$, for some $m \geq 1$, where once again, if $A$ is singular, the zero root is counted $n-\operatorname{rank}(A)$ times.

The examples in Giannoni and Woodford (2002) demonstrate that this can easily occur in models of practical interest; note that Assumption 7 is implied by the Sargent-Wallace property (that holds in our examples). In such a case, we obtain the following stronger characterization of optimal policy.

Proposition 3. In the case of a linear-quadratic policy problem satisfying Assumptions $1-7$, with $\lambda_{i}>0$, the optimal instrument rule (7.1) necessarily involves weights on lagged interest rates that are large enough for the lag polynomial $\alpha(L)=1-\gamma(L) L$ to have $m$ zeroes inside the unit circle. Let this polynomial be factored as

$$
\alpha(L)=\alpha_{1}(L) \alpha_{2}(L)
$$

where $\alpha_{1}(L)$ is a polynomial of order $m$ with all of its zeroes inside the unit circle and $\alpha_{2}(L)$ is a polynomial of order $k-m$ with all of its zeroes outside the unit circle. Then another optimal policy rule is given by

$$
\mathrm{E}_{t}\left\{\tilde{\alpha}_{1}\left(L^{-1}\right)^{-1} B_{2}^{\prime} B(L) \mathrm{E}_{t}\left[Q\left(L^{-1}\right) \hat{T}^{\prime} \hat{W}\left(\hat{\tau}_{t}-\hat{\tau}^{*}\right)\right]\right\}+\lambda_{i} \alpha_{2}(L)\left(i_{t-m}-i^{*}\right)=0
$$


where $\tilde{\alpha}_{1}\left(L^{-1}\right) \equiv \alpha_{1}(L) L^{-m}$. Note that this can only be interpreted as a targeting rule, since the criterion (7.5) does not involve the period $t$ instrument setting $i_{t}$. Rules (7.1) and (7.5) are equivalent in the sense that bounded processes $\left\{i_{t}\right\}$ and $\left\{\hat{\tau}_{t}\right\}$ satisfy (7.1) if and only if they satisfy (7.5). Thus (7.5) is an example of a robustly optimal direct targeting rule that cannot be interpreted as an instrument rule.

Note that the result that $\alpha(L)$ has zeroes inside the unit circle means that the instrument dynamics implied by (7.1) in the case of an arbitrary bounded path for the other target variables $\left\{\hat{\tau}_{t}\right\}$ will almost always be explosive. Thus the finding of Rotemberg and Woodford (1999) and Woodford (1999a), that an optimal interest-rate rule for certain forward-looking models involves "superinertial" interest-rate dynamics, is no fluke; for an interesting general class of forward-looking models, every optimal instrument rule of the form (7.1) will have this property.

Of course, commitment to such a rule does not imply that interest-rate dynamics will be explosive in equilibrium; to the contrary, we have shown that a rational-expectations equilibrium exists in which the interest rate (along with all of the other state variables) is bounded, and the fact that the dynamics are explosive in all other solutions to the system consisting of (2.1) and (7.1) is what makes this equilibrium determinate. In fact, under the assumption that private-sector expectations coordinate upon the determinate rationalexpectations equilibrium consistent with a given policy rule, a commitment to set interest rates according to a "superinertial" rule represents a way of forcing the evolution of the other target variables to satisfy the condition that is required in order for the implied interest-rate dynamics not to be explosive. The expectation that the evolution of the target variables will satisfy this condition is exactly what is expressed by the target criterion (7.5). Thus a commitment to set interest rates according to (7.1) and a commitment to adjust interest rates as necessary to ensure that criterion (7.5) holds at all times are not really different policies; the representation of policy in terms of an implicit instrument rule simply specifies more directly the size of interest-rate adjustments that are involved. 
The possibility of equivalent representations of optimal policy in terms of either an implicit instrument rule or a targeting rule is illustrated in Giannoni and Woodford (2002). Note that the equivalence of the two representations holds not only in the case of a single specification of the disturbance processes, but for all possible disturbance processes. Thus neither representation is more robust than the other, or requires more information for its implementation than does the other, or allows more scope for the exercise of "judgment" than does the other. We accordingly see little ground for drawing a sharp distinction between implicit instrument rules and targeting rules as general approaches to the conduct of monetary policy. If there are grounds for preferring one form of policy commitment to the other in a case of the sort described by Proposition 3, these presumably have to do with ease of communication with the public about commitments of the two types, rather than with any differences in the consequences associated with a credible commitment of either type. 


\section{References}

Batini, Nicoletta, and Andrew G. Haldane, "Forward-Looking Rules for Monetary Policy," in J.B. Taylor, ed., Monetary Policy Rules, Chicago: University of Chicago Press, 1999.

Batini, Nicoletta, and Joe Pearlman, "Too Much Too Soon: Instability and Indeterminacy with Forward-Looking Rules," unpublished, Bank of England, July 2002.

Black, Richard, Tiff Macklem, and David Rose, "On Policy Rules for Price Stability," in T. Macklem, ed., Price Stability, Inflation Targets and Monetary Policy, Ottawa: Bank of Canada, 1997.

Currie, David, and Paul Levine, Rules, Reputation and Macroeconomic Policy Coordination, Cambridge: Cambridge University Press, 1993.

Giannoni, Marc P., "Robust Optimal Monetary Policy in a Forward-Looking Model with Parameter and Shock Uncertainty," unpublished, Princeton University, 2001.

_- - - "Does Model Uncertainty Justify Caution? Robust Optimal Monetary Policy in a Forward-Looking Model," Macroeconomic Dynamics 6: 111-144 (2002).

_- - - and Michael Woodford, "Optimal Interest-Rate Rules: II. Applications," unpublished, Princeton University, August 2002.

Hansen, Lars P., and Thomas J. Sargent, "Robust Control and Filtering of ForwardLooking Models," unpublished, University of Chicago, October 2000.

Levin, Andrew, Volker Wieland, and John C. Williams, "The Performance of ForecastBased Monetary Policy Rules," unpublished, Federal Reserve Board, 2001.

McCallum, Bennett T., "Issues in the Design of Monetary Policy Rules," in J.B. Taylor and M. Woodford, eds., Handbook of Macroeconomics, vol. 1C, Amsterdam: North-Holland, 1999.

- - - , and Edward Nelson, "Timeless Perspective vs. Discretionary Monetary Policy in Forward-Looking Models," NBER Working Paper no. 7915, November 2000.

Rotemberg, Julio J., and Michael Woodford, "An Optimization-Based Econometric Frame- 
work for the Evaluation of Monetary Policy," NBER Macroeconomics Annual 12: 297-346 (1997).

- - - and - - - "Interest-Rate Rules in an Estimated Sticky-Price Model," in J.B. Taylor, ed., Monetary Policy Rules, Chicago: University of Chicago Press, 1999. Rudebusch, Glenn D., and Lars E.O. Svensson, "Policy Rules for Inflation Targeting," in J.B. Taylor, ed., Monetary Policy Rules, Chicago: University of Chicago Press, 1999.

Sargent, Thomas J., and Neil Wallace, "Rational' Expectations, the Optimal Monetary Instrument, and the Optimal Money Supply Rule," Journal of Political Economy 83: 241-254 (1975).

Svensson, Lars E.O., "Inflation Forecast Targeting: Implementing and Monitoring Inflation Targets," European Economic Review 41: 1111-1146 (1997).

_- - - "Inflation Targeting as a Monetary Policy Rule," Journal of Monetary Economics 43: 607-654 (1999a).

- - - "How Should Monetary Policy Be Conducted in an Era of Price Stability?" in New Challenges for Monetary Policy, Kansas City: Federal Reserve Bank of Kansas City, 1999b.

_- - - "What is Wrong with Taylor Rules? Using Judgment in Monetary Policy through Targeting Rules," unpublished, Princeton University, October 2001.

_- - - and Michael Woodford, "Implementing Optimal Policy through Inflation-Forecast Targeting," unpublished, Princeton University, November 1999.

_- - and - - - "Indicator Variables for Optimal Policy," NBER Working Paper no. 7953 , October 2000.

- - and - - " "Indicator Variables for Optimal Policy under Asymmetric Information," NBER Working Paper no. 8255, April 2001. (Forthcoming, Journal of Economic Dynamics and Control.)

Taylor, John B., "Discretion versus Policy Rules in Practice," Carnegie-Rochester Con- 
ference Series on Public Policy 39: 195-214 (1993).

- - - ed., Monetary Policy Rules, Chicago: University of Chicago Press, 1999.

Vickers, John, "Inflation Targeting in Practice: The U.K. Experience," Bank of England Quarterly Bulletin, November 1998.

Woodford, Michael, "Optimal Monetary Policy Inertia," NBER Working Paper no. 7261, August 1999a.

- _ - "Commentary: How Should Monetary Policy Be Conducted in an Era of Price Stability?" in New Challenges for Monetary Policy, Kansas City: Federal Reserve Bank of Kansas City, 1999b.

_- _-, "Pitfalls of Forward-Looking Monetary Policy," American Economic Review 90(2): 100-104 (2000).

- - - Interest and Prices: Foundations of a Theory of Monetary Policy, unpublished, Princeton University, July 2002. 


\section{A Proofs of Propositions}

\section{A.1 Assumptions 3 and 4}

The proof of Proposition 1 requires additional technical assumptions, beyond those stated in the text.

Assumption 3. The characteristic polynomial (2.3) has $\operatorname{rank}(\tilde{E})+\operatorname{rank}(A)-n_{z}$ distinct non-zero roots, in addition to the root zero if $\operatorname{rank}(A)<n$ (i.e., if $A$ is singular). In addition, if $\operatorname{rank}(A)<n$, there are $n-\operatorname{rank}(A)$ linearly independent vectors $u_{i}$ such that $A u_{i}=0$, and likewise $n-\operatorname{rank}(A)$ linearly independent vectors $f_{i}^{\prime}$ such that $f_{i}^{\prime} A=0$. Similarly, if $\operatorname{rank}(\tilde{E})<n_{z}$, there are $n_{z}-\operatorname{rank}(\tilde{E})$ linearly independent vectors $e_{j}^{\prime}$ such that $e_{j}^{\prime} \hat{I}=0$, and likewise $n_{z}-\operatorname{rank}(\tilde{E})$ linearly independent vectors $h_{j}$ such that $\hat{I} h_{j}=0$.

Assumption 3 implies that there $\operatorname{are} \operatorname{rank}(\hat{I})=n_{Z}+\operatorname{rank}(\tilde{E})$ linearly independent vectors $u_{i}$ with the property that

$$
A u_{i}=\mu_{i} \hat{I} u_{i}
$$

for some (scalar) eigenvalue $\mu_{i}$. The eigenvalues correspond to the roots $\mu_{i}$ of (2.3), and there is one eigenvector for each of the non-zero roots, in addition to $n-\operatorname{rank}(A)$ corresponding to the zero root. The eigenvectors associated with real eigenvalues are real-valued, while the eigenvectors associated with complex eigenvalues are complex-valued. Complex roots come in complex conjugate pairs, and if $u_{i}$ is the eigenvector associated with a complex eigenvalue $\mu_{i}$, then the eigenvector associated with $\mu_{i}^{\dagger}$ is $u_{i}^{\dagger}$, where the dagger denotes a complex conjugate. There is also an additional set of $n_{z}-\operatorname{rank}(\tilde{E})$ linearly independent vectors $h_{j}$ such that $\hat{I} h_{j}=0$, necessarily linearly independent of the set of eigenvectors. Taken together, these vectors comprise a set of $n$ linearly independent vectors that can be used as a basis for $\mathcal{C}^{n}$.

There are similarly at least $\operatorname{rank}(A)$ linearly independent vectors $e_{j}^{\prime}$ with the property 
that

$$
\theta_{j} e_{j}^{\prime} A=e_{j}^{\prime} \hat{I}
$$

for some $\theta_{j} \cdot{ }^{31}$ Corresponding to each of the non-zero roots $\mu_{i}$ of (2.3) there is a left eigenvector $e_{i}^{\prime}$ satisfying this equation for $\theta_{i}=\mu_{i}^{-1}$. The remaining left eigenvectors are the $n_{z}-\operatorname{rank}(\tilde{E})$ linearly independent vectors $e_{j}^{\prime}$ such that $e_{j}^{\prime} \hat{I}=0$; these satisfy (A.2) for $\theta_{j}=0$. Again there is also an additional set of $n-\operatorname{rank}(A)$ linearly independent vectors $f_{i}^{\prime}$ such that $f_{i}^{\prime} A=0$. Taken together, this set of $n$ generalized left eigenvectors also forms a basis for $\mathcal{C}^{n}$.

In the case of a left eigenvector $e_{j}^{\prime}$ and a right eigenvector $u_{i}$ corresponding to eigenvalues such that $\theta_{j} \mu_{i} \neq 1$, we necessarily have

$$
e_{j}^{\prime} A u_{i}=e_{j}^{\prime} \hat{I} u_{i}=0
$$

The same condition can be shown to hold if either $e_{j}^{\prime}$ or $u_{i}$ is one of the generalized eigenvectors, i.e., if $e_{j}^{\prime} A=0$ or $\hat{I} u_{i}=0$. We can also normalize the eigenvectors so that if $e_{j}^{\prime}$ and $u_{i}$ are eigenvectors for which $\theta_{j} \mu_{i}=1$, then

$$
e_{j}^{\prime} \hat{I} u_{i}=1
$$

Assumptions 2 and 3 together imply that there exist at least $n_{Z}$ linearly independent vectors $u_{i}$ satisfying (A.1) for which the associated eigenvalue satisfies $\left|\mu_{i}\right|<\beta^{-1}$. Thus we can form a $n \times n_{Z}$ matrix $J$ with the property that

$$
A J=\hat{I} J \Omega
$$

where $\Omega$ is a square matrix of dimension $n_{Z}$, all of the eigenvalues of which satisfy $\left|\mu_{i}\right|<\beta^{-1}$. This last property implies that

$$
\|\beta \Omega\|<1
$$

The columns of $J$ are linear combinations of $n_{Z}$ of the eigenvectors $u_{i}$, and the eigenvalues of $\Omega$ are the associated eigenvalues. ${ }^{32}$ It is possible to include all of the vectors $u_{i}$ such that

\footnotetext{
${ }^{31}$ Note that in the case of a complex-valued column vector or matrix $e$, we use the notation $e^{\prime}$ to denote the transpose of $e$, not the conjugate transpose.

${ }^{32}$ In the case that one selects a complex conjugate pair of eigenvectors, it is convenient for the columns of $J$ not to be the eigenvectors themselves, so that the columns of $J$ can be real-valued.
} 
$A u_{i}=0$ among the eigenvectors used to form the columns of $J$, and we shall assume that $J$ is constructed in this way.

When it is possible to select a set of eigenvectors that are all real-valued, or that consist of real-valued eigenvectors along with one or more complex conjugate pairs (case (i) of Assumption 2), it is possible to choose the matrices $J$ and $\Omega$ to be real-valued. However, if the non-zero eigenvalues such that $\left|\mu_{i}\right|<\beta^{-1}$ are all complex and $\operatorname{rank}(A)-n_{z}$ is odd, then it is necessary to split a complex conjugate pair, and in this case both $J$ and $\Omega$ must be complex-valued. ${ }^{33}$ Even in this case, however, it is possible to choose $J$ and $\Omega$ to be of the form

$$
J=\left[\begin{array}{ll}
\tilde{J} & u
\end{array}\right], \quad \Omega=\left[\begin{array}{cc}
\tilde{\Omega} & 0 \\
0 & \mu
\end{array}\right],
$$

where $\tilde{J}$ and $\tilde{\Omega}$ are real-valued, and $u$ is the complex-valued right eigenvector (i.e., vector satisfying (A.1)) corresponding to the complex eigenvalue $\mu$. In this case (case (ii) of Assumption 2), we have assumed that the eigenvalue $\mu$ is such that $\left|\operatorname{Re} \mu^{-1}\right|>\beta$. We shall assume that $J$ and $\Omega$ are constructed in one of these two ways.

Corresponding to this selection from among the right eigenvectors, we may similarly form two sets of (generalized) left eigenvectors, $\Phi$ and $\Psi$. Here the rows of $\Psi^{\prime}$ are $n_{z}$ linearly independent combinations of the $n_{z}$ left eigenvectors with eigenvalues equal to zero, or to the reciprocals of roots of (2.3) that are not among the eigenvalues of $\Omega$. It follows that there is a matrix $\Theta$ such that

$$
\Theta^{\prime} \Psi^{\prime} A=\Psi^{\prime} \hat{I}
$$

this matrix is of $\operatorname{rank} k \equiv \operatorname{rank}(\tilde{E})$ and has non-zero eigenvalues corresponding to the reciprocals of the roots of (2.3) just mentioned. It also follows from (A.3) that

$$
\Psi^{\prime} A J=\Psi^{\prime} \hat{I} J=0
$$

Similarly, the rows of $\Phi^{\prime}$ are $n_{Z}$ linear combinations of the $n-\operatorname{rank}(A)$ vectors $f_{i}^{\prime}$ such that $f_{i}^{\prime} A=0$ and the $\operatorname{rank}(A)-n_{z}$ left eigenvectors with eigenvalues that are the reciprocals

\footnotetext{
${ }^{33}$ This happens in the example in Giannoni and Woodford (2002) with inflation inertia, in the case that the degree of inertia is large.
} 
of non-zero eigenvalues of $\Omega$. We can select this matrix so that

$$
\begin{gathered}
\Phi^{\prime} A=\Omega \Phi^{\prime} \hat{I}, \\
\Phi^{\prime} \hat{I} J=I .
\end{gathered}
$$

Note that the columns of $\Psi$ and $\Phi$ together form a basis for $\mathcal{C}^{n}$.

In case (i) of Assumption 2, the matrices $\Psi, \Phi$ and $\Theta$ are all real-valued. On the other hand, in case (ii), when a complex pair of eigenvalues is split in constructing $J$ and $\Omega$, it is also necessary for $\Psi$ and $\Phi$ to each contain at least one complex-valued eigenvector, and for $\Theta$ to have at least one complex eigenvalue. We can, however, choose these matrices to be of the form

$$
\begin{aligned}
& \Psi=\left[\begin{array}{ll}
\tilde{\Psi} & v
\end{array}\right], \quad \Theta=\left[\begin{array}{cc}
\tilde{\Theta} & 0 \\
0 & \mu^{\dagger-1}
\end{array}\right], \\
& \Phi=\left[\begin{array}{ll}
\tilde{\Phi} & v^{\dagger}
\end{array}\right],
\end{aligned}
$$

where $\tilde{\Psi}, \tilde{\Theta}$, and $\tilde{\Phi}$ are all real-valued. Here $\mu$ is again the complex eigenvalue in (A.6), $v^{\prime}$ is the complex-valued left eigenvector associated with eigenvalue $\mu^{\dagger-1}$, and the dagger denotes the complex conjugate. We shall assume that in case (ii) the matrices $\Psi, \Phi$ and $\Theta$ are of this form.

We can now state our remaining technical assumption.

Assumption 4. The matrix of left eigenvectors $\Psi$ is such that (i) the matrix $A^{\prime} \Psi$ is of full rank (i.e., rank $n_{z}$ ); and (ii) if the rows of $\Psi$ are partitioned

$$
\Psi \equiv\left[\begin{array}{l}
\Psi_{1} \\
\Psi_{2}
\end{array}\right],
$$

conformably with the partition of the vector of endogenous variables in (2.1), then

$$
\operatorname{det}\left[\operatorname{Re} \Psi_{2}\right] \neq 0 \text {. }
$$

Like Assumption 3, this assumption will be satisfied by generic matrices $A$ and $\tilde{E}$ of arbitrary $\operatorname{ranks} 0 \leq \operatorname{rank}(\tilde{E}) \leq n_{z} \leq \operatorname{rank}(A) \leq n$. We turn now to the proofs of the Propositions in the text. 


\section{A.2 Proof of Proposition 1}

Proposition 1. Under Assumptions $2-4$, there exist (real-valued) matrices $\Lambda$ and $\Upsilon$ and linear operators $Q\left(L^{-1}\right)$ and $R\left(L^{-1}\right)$ such that bounded processes $\left\{\varphi_{t}\right\}$ and $\left\{\tau_{t}\right\}$ satisfy (6.1) for all $t \geq t_{0}$ if and only if they satisfy the conditions

$$
\begin{aligned}
\Xi_{t} & =\Lambda \Xi_{t-1}+\mathrm{E}_{t}\left[Q\left(L^{-1}\right) \tilde{T}^{\prime} W\left(\tau_{t}-\tau^{*}\right)\right] \\
\xi_{t} & =\Upsilon \Xi_{t-1}+\mathrm{E}_{t}\left[R\left(L^{-1}\right) \tilde{T}^{\prime} W\left(\tau_{t}-\tau^{*}\right)\right]
\end{aligned}
$$

for all $t \geq t_{0}$. These linear operators are such that $Q\left(L^{-1}\right) x_{t}$ and $R\left(L^{-1}\right) x_{t}$ are well-defined and bounded processes in the case of any bounded process $x_{t}$.

ProOF: Let $J$ be a matrix satisfying (A.4) for some matrix $\Omega$ with the properties stated above. Then premultiplying (6.1) by $J^{\prime}$, using the transpose of (A.4) to substitute for $J^{\prime} A^{\prime}$, and re-arranging terms, we obtain

$$
\mathrm{E}_{t}\left[\left(I-\beta \Omega^{\prime} L^{-1}\right) J^{\prime} \hat{I}^{\prime} \varphi_{t}\right]=\beta J^{\prime} \tilde{T}^{\prime} W\left(\tau_{t}-\tau^{*}\right)
$$

Because of (A.5), $\left(I-\beta \Omega^{\prime} L^{-1}\right)$ is invertible, and the above is equivalent to

$$
J^{\prime} \hat{I}^{\prime} \varphi_{t}=\beta \mathrm{E}_{t}\left[\left(I-\beta \Omega^{\prime} L^{-1}\right)^{-1} J^{\prime} \tilde{T}^{\prime} W\left(\tau_{t}-\tau^{*}\right)\right]
$$

in the sense that bounded processes $\left\{\varphi_{t}, \tau_{t}\right\}$ satisfy one relation for all $t \geq t_{0}$ if and only if they satisfy the other.

Now let the columns of $\Psi$ and $\Phi$ be used as a basis, and represent $\varphi_{t}$ in the form

$$
\varphi_{t} \equiv \Psi \psi_{t}+\Phi \phi_{t}
$$

Using (A.8) and (A.10), it follows that $J^{\prime} \hat{I}^{\prime} \varphi_{t}=\phi_{t}$, so that (A.15) provides a solution for the coefficients $\phi_{t}$ in the case of any bounded process for the evolution of the target variables.

Substituting (A.16) into (6.1) to eliminate $\varphi_{t}$, and then substituting the right-hand side of (A.15) for $\phi_{t}$, we obtain

$$
A^{\prime} \Psi \mathrm{E}_{t} \psi_{t+1}=\beta^{-1} \hat{I}^{\prime} \Psi \psi_{t}+\left(\hat{I}^{\prime} \Phi J^{\prime}-I\right) \tilde{T}^{\prime} W\left(\tau_{t}-\tau^{*}\right)
$$


as a restriction upon the optimal evolution of the coefficients $\left\{\psi_{t}\right\}$. (Here we have used (A.9) to substitute for $A^{\prime} \Phi$.)

We note furthermore that (A.10) implies that

$$
J^{\prime}\left(\hat{I}^{\prime} \Phi J^{\prime}-I\right)=0
$$

But (A.8), together with part (i) of Assumption 4, implies that the $2 n_{z}$-dimensional subspace of $\mathcal{C}^{n}$ consisting of vectors orthogonal to all rows of $J^{\prime}$ is spanned by the columns of $A^{\prime} \Psi$. Thus there must be a representation of the form

$$
\hat{I}^{\prime} \Phi J^{\prime}-I=A^{\prime} \Psi S
$$

for some matrix $S$. Substituting this, and also using (A.7) to substitute for $\hat{I}^{\prime} \Psi$, condition (A.17) may be written

$$
A^{\prime} \Psi\left[\mathrm{E}_{t} \psi_{t+1}-\beta^{-1} \Theta \psi_{t}\right]=A^{\prime} \Psi S \tilde{T}^{\prime} W\left(\tau_{t}-\tau^{*}\right)
$$

Finally, because $A^{\prime} \Psi$ is of full rank, this is possible only if

$$
\mathrm{E}_{t} \psi_{t+1}=\beta^{-1} \Theta \psi_{t}+S \tilde{T}^{\prime} W\left(\tau_{t}-\tau^{*}\right)
$$

\section{A.2.1 Case (i)}

Let us now consider first case (i) of Assumption 2, in which case $J, \Psi$ and $\Phi$ are all realvalued. If we partition the rows of $\Phi$ in the same way as those of $\Psi$, then (A.16) implies that

$$
\Xi_{t-1}=\Psi_{2} \psi_{t}+\Phi_{2} \phi_{t}
$$

and part (ii) of Assumption 4 implies that $\Psi_{2}$ is invertible, allowing us to solve for

$$
\psi_{t}=\Psi_{2}^{-1} \Xi_{t-1}-\Psi_{2}^{-1} \Phi_{2} \phi_{t}
$$

Substituting this into (A.18) to eliminate $\psi_{t}$, then substituting the right-hand side of (A.15) for $\phi_{t}$, and finally premultiplying all terms by $\Psi_{2}$, we obtain a relation of the form (A.13), in which

$$
\Lambda \equiv \beta^{-1} \Psi_{2} \Theta \Psi_{2}^{-1}
$$




$$
Q\left(L^{-1}\right) \equiv \beta\left(L^{-1}-\Lambda\right) \Phi_{2}\left(I-\beta \Omega^{\prime} L^{-1}\right)^{-1} J^{\prime}+\Psi_{2} S
$$

Similarly, (A.16) implies that

$$
\xi_{t}=\Psi_{1} \psi_{t}+\Phi_{1} \phi_{t}
$$

Substituting (A.20) for $\psi_{t}$ and (A.15) for $\phi_{t}$, we obtain a relation of the form (A.14), in which

$$
\begin{gathered}
\Upsilon \equiv \Psi_{1} \Psi_{2}^{-1} \\
R\left(L^{-1}\right) \equiv \beta\left(\Phi_{1}-\Psi_{1} \Psi_{2}^{-1} \Phi_{2}\right)\left(I-\beta \Omega^{\prime} L^{-1}\right)^{-1} J^{\prime} .
\end{gathered}
$$

Note that in this case $\Lambda, \Upsilon, Q\left(L^{-1}\right)$ and $R\left(L^{-1}\right)$ are all real-valued.

We have shown that with these definitions, any bounded processes $\left\{\varphi_{t}\right\}$ and $\left\{\tau_{t}\right\}$ that satisfy (6.1) for all $t \geq t_{0}$ must also satisfy (A.13) - (A.14) for all $t \geq t_{0}$. Conversely, suppose that one is given bounded processes that satisfy the latter conditions each period. Then defining $\psi_{t}$ by (A.20) and $\phi_{t}$ by the right-hand side of (A.15), using (A.13) one can show that the process $\left\{\psi_{t}\right\}$ must satisfy (A.18), and hence (A.17) for all $t \geq t_{0}$. Using the definition of $\phi_{t}$ one can then write this in the form

$$
A^{\prime}\left(\Psi \mathrm{E}_{t} \psi_{t+1}+\Phi \mathrm{E}_{t} \phi_{t+1}\right)=\beta^{-1} \hat{I}\left(\Psi \psi_{t}+\Phi \phi_{t}\right)-\tilde{T}^{\prime} W\left(\tau_{t}-\tau^{*}\right)
$$

Similarly, one observes that (A.14) implies (A.22), while the definition of $\psi_{t}$ implies (A.19). Together these relations imply that $\varphi_{t}$ satisfies (A.16). But using this one observes that (A.23) implies (6.1), so that this relation must hold for all $t \geq t_{0}$. Thus the proposition is established, for case (i) of Assumption 2.

\section{A.2.2 Case (ii)}

Now consider instead case (ii), in which $J, \Psi$ and $\Phi$ each have one complex-valued column.

In this case, in the representation (A.16), the coefficients $\psi_{t}$ and $\phi_{t}$ are complex-valued. However, if we introduce the decompositions

$$
\psi_{t} \equiv\left[\begin{array}{c}
\tilde{\psi}_{t} \\
\psi_{v t}
\end{array}\right], \quad \phi_{t} \equiv\left[\begin{array}{c}
\tilde{\phi}_{t} \\
\phi_{v t}
\end{array}\right]
$$


where in each case the lower sub-vector has only a single element, then $\tilde{\psi}_{t}$ and $\tilde{\phi}_{t}$ will be real-valued, and the two complex elements will satisfy $\phi_{v t}=\psi_{v t}^{\dagger}$.

Furthermore, using the previously introduced decompositions of $J$ and $\Omega$, the right-hand side of (A.15) can be decomposed to yield the two equations

$$
\begin{aligned}
\tilde{\phi}_{t} & =\beta \mathrm{E}_{t}\left[\left(I-\beta \tilde{\Omega}^{\prime} L^{-1}\right)^{-1} \tilde{J}^{\prime} \tilde{T}^{\prime} W\left(\tau_{t}-\tau^{*}\right)\right], \\
\phi_{v t} & =\beta \mathrm{E}_{t}\left[\left(1-\beta \mu L^{-1}\right)^{-1} u^{\prime} \tilde{T}^{\prime} W\left(\tau_{t}-\tau^{*}\right)\right] .
\end{aligned}
$$

We note that all coefficients appearing in (A.24) are real-valued, so that the equation implies a real-valued bounded process for $\left\{\tilde{\phi}_{t}\right\}$ corresponding to any real-valued bounded process for $\left\{\tau_{t}\right\}$.

Similarly, each of the terms in (A.17) can be decomposed, yielding

$$
\begin{aligned}
A^{\prime} \tilde{\Psi} \mathrm{E}_{t} \tilde{\psi}_{t+1}+A^{\prime} v \mathrm{E}_{t} \psi_{v, t+1} & =\beta^{-1} A^{\prime} \tilde{\Psi} \tilde{\Theta} \tilde{\psi}_{t}+\beta^{-1} \mu^{\dagger-1} A^{\prime} v \psi_{v t} \\
& +\left[\left(\hat{I}^{\prime} \tilde{\Phi} \tilde{J}^{\prime}-I\right)+\mu^{-1} A^{\prime} v^{\dagger} u^{\prime}\right] \tilde{T}^{\prime} W\left(\tau_{t}-\tau^{*}\right) .
\end{aligned}
$$

The fact that $\hat{I}^{\prime} \tilde{\Phi} \tilde{J}^{\prime}-I$ is real-valued and satisfies

$$
\tilde{J}^{\prime}\left(\hat{I}^{\prime} \tilde{\Phi} \tilde{J}^{\prime}-I\right)=0
$$

implies that it must have a representation of the form

$$
\hat{I}^{\prime} \tilde{\Phi} \tilde{J}^{\prime}-I=A^{\prime} \tilde{\Psi} \tilde{S}+A^{\prime} v s^{\prime}+A^{\prime} v^{\dagger} s^{\dagger \prime}
$$

for some real-valued matrix $\tilde{S}$ and complex-valued vector $s^{\prime}$. The fact that in addition

$$
u^{\prime}\left(\hat{I}^{\prime} \tilde{\Phi} \tilde{J}^{\prime}-I\right)=-u^{\prime}
$$

then implies furthermore that $s^{\prime}=-\mu^{\dagger-1} u^{\dagger}$. Hence equations (A.18) can be separated, in this case, into two equations,

$$
\begin{aligned}
\mathrm{E}_{t} \tilde{\psi}_{t+1} & =\beta^{-1} \tilde{\Theta} \tilde{\psi}_{t}+\tilde{S} \tilde{T}^{\prime} W\left(\tau_{t}-\tau^{*}\right) \\
\mathrm{E}_{t} \psi_{v, t+1} & =\beta^{-1} \mu^{\dagger-1} \psi_{v t}-\mu^{\dagger-1} u^{\dagger \prime} \tilde{T}^{\prime} W\left(\tau_{t}-\tau^{*}\right)
\end{aligned}
$$


corresponding to the coefficients multiplying $A^{\prime} \tilde{\Psi}$ and those multiplying $A^{\prime} v$, respectively. We note that all of the coefficients appearing in (A.27) are real-valued.

Finally, we note that (A.28) may equivalently be written

$$
\mathrm{E}_{t}\left[\left(1-\beta \mu^{\dagger} L^{-1}\right) \psi_{v t}\right]=\beta u^{\dagger \prime} \tilde{T}^{\prime} W\left(\tau_{t}-\tau^{*}\right) .
$$

Because $|\mu|<\beta^{-1}$, the operator $1-\beta \mu^{\dagger} L^{-1}$ is invertible, and this relation is equivalent to

$$
\psi_{v t}=\beta \mathrm{E}_{t}\left[\left(1-\beta \mu^{\dagger} L^{-1}\right)^{-1} u^{\dagger \prime} \tilde{T}^{\prime} W\left(\tau_{t}-\tau^{*}\right)\right] .
$$

Comparison of this relation with (A.25) indicates that these relations imply that $\phi_{v t}=\psi_{v t}^{\dagger}$, as is necessary in order for $\varphi_{t}$ to be real-valued.

It is useful now to introduce an alternative set of basis vectors, a set of $n$ real-valued vectors that span $\mathcal{R}^{n}$, consisting of the columns of the matrices $\bar{\Psi}$ and $\bar{\Phi}$ defined by

$$
\bar{\Psi} \equiv\left[\begin{array}{ll}
\tilde{\Psi} & \bar{v}
\end{array}\right], \quad \bar{\Phi} \equiv\left[\begin{array}{ll}
\tilde{\Phi} & \tilde{v}
\end{array}\right],
$$

where $\bar{v} \equiv \operatorname{Re} v$ and $\tilde{v} \equiv \operatorname{Im} v$. In terms of this new basis, $\varphi_{t}$ can be given the representation

$$
\varphi_{t}=\bar{\Psi} \bar{\psi}_{t}+\bar{\Phi} \bar{\phi}_{t}
$$

where

$$
\bar{\psi}_{t} \equiv\left[\begin{array}{c}
\tilde{\psi}_{t} \\
\psi_{r t}
\end{array}\right], \quad \bar{\phi}_{t} \equiv\left[\begin{array}{c}
\tilde{\phi}_{t} \\
\phi_{i t}
\end{array}\right],
$$

and

$$
\psi_{r t} \equiv 2 \operatorname{Re} \psi_{v t}, \quad \phi_{i t} \equiv 2 \operatorname{Im} \phi_{v t} .
$$

Note that now all of the coefficients $\bar{\psi}_{t}$ and $\bar{\phi}_{t}$ are real-valued.

Given the definitions of the new coefficient $\phi_{i t}$, (A.25) implies that

$$
\begin{aligned}
\phi_{i t} & =\frac{\beta}{i} \mathrm{E}_{t}\left\{\left[\left(1-\beta \mu L^{-1}\right)^{-1} u^{\prime}-\left(1-\beta \mu^{\dagger} L^{-1}\right)^{-1} u^{\dagger}\right] \tilde{T}^{\prime} W\left(\tau_{t}-\tau^{*}\right)\right\} \\
& \equiv \mathrm{E}_{t}\left[f\left(L^{-1}\right)^{\prime} \tilde{T}^{\prime} W\left(\tau_{t}-\tau^{*}\right)\right] .
\end{aligned}
$$

Here all of the coefficients of $f\left(L^{-1}\right)$ are real-valued. Hence the complete vector of coefficients $\bar{\phi}_{t}$ is given by

$$
\bar{\phi}_{t}=\mathrm{E}_{t}\left[F\left(L^{-1}\right) \tilde{T}^{\prime} W\left(\tau_{t}-\tau^{*}\right)\right],
$$


where

$$
F\left(L^{-1}\right) \equiv\left[\begin{array}{c}
\beta\left(I-\beta \tilde{\Omega}^{\prime} L^{-1}\right)^{-1} \tilde{J}^{\prime} \\
f\left(L^{-1}\right)^{\prime}
\end{array}\right] .
$$

Similarly, given the definition of $\psi_{r t}$, (A.28) implies that

$$
\begin{aligned}
\mathrm{E}_{t} \psi_{r, t+1} & =\beta^{-1} \operatorname{Re}\left\{\mu^{\dagger-1}\left(\psi_{r t}-i \phi_{i t}\right)\right\}-2 \operatorname{Re}\left\{\mu^{\dagger-1} u^{\dagger \prime}\right\} \tilde{T}^{\prime} W\left(\tau_{t}-\tau^{*}\right), \\
& =\bar{\lambda} \psi_{r t}+\tilde{\lambda} \phi_{i t}-\left(\mu^{-1} u^{\prime}+\mu^{\dagger-1} u^{\dagger \prime}\right) \tilde{T}^{\prime} W\left(\tau_{t}-\tau^{*}\right), \\
& =\bar{\lambda} \psi_{r t}+\mathrm{E}_{t}\left[g\left(L^{-1}\right)^{\prime} \tilde{T}^{\prime} W\left(\tau_{t}-\tau^{*}\right)\right],
\end{aligned}
$$

where

$$
\bar{\lambda} \equiv \beta^{-1} \operatorname{Re} \mu^{\dagger-1}, \quad \tilde{\lambda} \equiv \beta^{-1} \operatorname{Im} \mu^{\dagger-1}
$$

and

$$
g\left(L^{-1}\right)^{\prime} \equiv \tilde{\lambda} f\left(L^{-1}\right)^{\prime}-\left(\mu^{-1} u^{\prime}+\mu^{\dagger-1} u^{\dagger \prime}\right)
$$

Here $\bar{\lambda}$ and all of the coefficients of $g\left(L^{-1}\right)^{\prime}$ are real-valued. Hence the complete vector of coefficients $\bar{\psi}_{t}$ satisfies a relation of the form

$$
\mathrm{E}_{t} \bar{\psi}_{t+1}=\beta^{-1} \bar{\Theta} \bar{\psi}_{t}+\mathrm{E}_{t}\left[G\left(L^{-1}\right) \tilde{T}^{\prime} W\left(\tau_{t}-\tau^{*}\right)\right]
$$

where $\bar{\Theta} \equiv \operatorname{Re} \Theta$ and

$$
G\left(L^{-1}\right) \equiv\left[\begin{array}{c}
\tilde{S} \\
g\left(L^{-1}\right)^{\prime}
\end{array}\right]
$$

Finally, if we partition the rows of $\bar{\Psi}$ and $\bar{\Phi}$ in the same way as before, and note that $\bar{\Psi} \equiv \operatorname{Re} \Psi$, we see that part (ii) of Assumption 4 implies that $\bar{\Psi}_{2}$ is non-singular. Then (A.30) implies that

$$
\bar{\psi}_{t}=\bar{\Psi}_{2}^{-1} \Xi_{t-1}-\bar{\Psi}_{2}^{-1} \bar{\Phi}_{2} \bar{\phi}_{t}
$$

analogous to (A.20) in the case of only real-valued eigenvectors. Substituting this into (A.34) to eliminate $\bar{\psi}_{t}$, and then substituting (A.32) for $\bar{\phi}_{t}$, we once again obtain a relation of the form (A.13), in which now

$$
\begin{gathered}
\Lambda \equiv \beta^{-1} \bar{\Psi}_{2} \bar{\Theta} \bar{\Psi}_{2}^{-1} \\
Q\left(L^{-1}\right) \equiv \bar{\Psi}_{2} G\left(L^{-1}\right)+\left(L^{-1}-\Lambda\right) \bar{\Phi}_{2} F\left(L^{-1}\right) .
\end{gathered}
$$


Similarly, (A.30) allows us to solve for $\xi_{t}$ as a function of $\Xi_{t-1}$ and $\bar{\phi}_{t}$. Substitution of (A.32) for $\bar{\phi}_{t}$ again yields a relation of the form (A.14), in which now

$$
\begin{gathered}
\Upsilon \equiv \bar{\Psi}_{1} \bar{\Psi}_{2}^{-1}, \\
R\left(L^{-1}\right) \equiv\left(\bar{\Phi}_{1}-\bar{\Psi}_{1} \bar{\Psi}_{2}^{-1} \bar{\Phi}_{2}\right) F\left(L^{-1}\right) .
\end{gathered}
$$

Once again, $\Lambda, \Upsilon, Q\left(L^{-1}\right)$ and $R\left(L^{-1}\right)$ are all real-valued.

Thus with these definitions, we have established that any bounded processes $\left\{\varphi_{t}\right\}$ and $\left\{\tau_{t}\right\}$ that satisfy (6.1) for all $t \geq t_{0}$ must also satisfy (A.13) - (A.14) for all $t \geq t_{0}$. The converse can also be established, as before, largely by reversing the series of calculations used to derive this result. Given bounded processes that satisfy (A.13) - (A.14) each period, one can define $\bar{\psi}_{t}$ by (A.35) and $\bar{\phi}_{t}$ by (A.32). It then follows that $\varphi_{t}$ satisfies (A.30) each period, where the coefficients $\bar{\psi}_{t}$ satisfy (A.34) and the coefficients $\bar{\phi}_{t}$ satisfy (A.32).

The one point at which our previous calculations are not directly reversible is where we derived the properties of the real-valued coefficients $\psi_{r t}$ and $\phi_{i t}$ from those of the complexvalued $\psi_{v t}$ and $\phi_{v t}$. While it is easy to see that (A.25) implies (A.31) and that (A.28) implies (A.33), the converses need not hold. Nonetheless, the fact that both (A.31) and (A.33) hold implies that (A.25) and (A.28) must hold. For (A.31) implies that

$$
\begin{aligned}
\mathrm{E}_{t}[(1-\beta \mu & \left.\left.L^{-1}\right)\left(1-\beta \mu^{\dagger} L^{-1}\right)\left(L^{-1}-\bar{\lambda}\right) \phi_{i t}\right] \\
\quad= & \mathrm{E}_{t}\left[\left(1-\beta \mu L^{-1}\right)\left(1-\beta \mu^{\dagger} L^{-1}\right)\left(L^{-1}-\bar{\lambda}\right) f\left(L^{-1}\right)^{\prime} \tilde{T}^{\prime} W\left(\tau_{t}-\tau^{*}\right)\right] \\
= & -2 \beta \operatorname{Im} \mathrm{E}_{t}\left[\left(L^{-1}-\bar{\lambda}\right)\left(1-\beta \mu L^{-1}\right) u^{\dagger \prime} \tilde{T}^{\prime} W\left(\tau_{t}-\tau^{*}\right)\right] .
\end{aligned}
$$

Similarly, (A.33) implies that

$$
\begin{aligned}
\mathrm{E}_{t}\left[\left(1-\beta \mu L^{-1}\right)\right. & \left.\left(1-\beta \mu^{\dagger} L^{-1}\right)\left(L^{-1}-\bar{\lambda}\right) \psi_{r t}\right] \\
& =\mathrm{E}_{t}\left[\left(1-\beta \mu L^{-1}\right)\left(1-\beta \mu^{\dagger} L^{-1}\right) g\left(L^{-1}\right)^{\prime} \tilde{T}^{\prime} W\left(\tau_{t}-\tau^{*}\right)\right] \\
& =2 \beta \operatorname{Re} \mathrm{E}_{t}\left[\left(L^{-1}-\bar{\lambda}\right)\left(1-\beta \mu L^{-1}\right) u^{\dagger \prime} \tilde{T}^{\prime} W\left(\tau_{t}-\tau^{*}\right)\right]
\end{aligned}
$$


Then, since

$$
\psi_{v t}=\frac{1}{2}\left(\psi_{r t}-i \phi_{i t}\right)
$$

it follows that

$$
\begin{aligned}
\mathrm{E}_{t}\left[\left(1-\beta \mu L^{-1}\right)\right. & \left.\left(1-\beta \mu^{\dagger} L^{-1}\right)\left(L^{-1}-\bar{\lambda}\right) \psi_{v t}\right] \\
& =\beta \mathrm{E}_{t}\left[\left(L^{-1}-\bar{\lambda}\right)\left(1-\beta \mu L^{-1}\right) u^{\dagger \prime} \tilde{T}^{\prime} W\left(\tau_{t}-\tau^{*}\right)\right] .
\end{aligned}
$$

Assumption 2 implies both that $|\mu|<\beta^{-1}$ and that $|\bar{\lambda}|>1$. (It is at this point that we require the assumption that $\left|\operatorname{Re} \mu^{-1}\right|>\beta$.) Hence the operators $1-\beta \mu L^{-1}, 1-\beta \mu^{\dagger} L^{-1}$, and $L^{-1}-\bar{\lambda}$ are each invertible, and (A.37) implies (A.29). This last equation then implies (A.28), and also (A.25), using the fact that $\phi_{v t}=\psi_{v t}^{\dagger}$. Thus we can show that the coefficients $\phi_{t}$ must satisfy (A.24) - (A.25), and that the coefficients $\psi_{t}$ must satisfy (A.18). It then follows as above that the processes $\left\{\varphi_{t}\right\}$ and $\left\{\tau_{t}\right\}$ must satisfy (6.1) for all $t \geq t_{0}$, and the proposition is established in case (ii) as well.

\section{A.2.3 Remarks}

We have noted in the text that there may be some arbitrariness in the construction of the $\Lambda, \Upsilon, Q$ and $R$ referred to in Proposition 1. This follows from the possible non-uniqueness of the set of $n_{Z}$ eigenvalues used to construct the matrices $J$ and $\Omega$. However, given a choice of $n_{Z}$ roots of (2.3) with the assumed properties, the choice of how to order the columns of $J$ can be shown not to matter for the $\Lambda, \Upsilon, Q$ and $R$ obtained from the above construction. Thus in the case that the number of roots such that $\left|\mu_{i}\right|<\beta^{-1}$ is exactly $n_{Z}$, the $\Lambda, \Upsilon, Q$ and $R$ referred to in Proposition 1 are uniquely determined.

Even when the number of such roots exceeds $n_{Z}$, there is an attractive method for selecting a particular characterization of the form given in Proposition 1. This is to form the matrix $J$ from the eigenvectors associated with a set of $n_{Z}$ roots that are chosen so as to minimize $\|\Lambda\|$, the upper bound on the eigenvalues of $\Lambda$; we shall call this the minimally inertial specification. It follows from (A.36) that the eigenvalues of $\Lambda$ are the set of quantities $\beta^{-1} \theta_{j}$, where $\theta_{j}$ is an eigenvalue of $\bar{\Theta} \equiv \operatorname{Re} \Theta$. The eigenvalues of $\Theta$, other than zero (in the 
case that $\tilde{E}$ is singular), are equal to the reciprocals of the $\operatorname{rank}(\tilde{E}) \operatorname{roots}$ of $(2.3)$ that are not included among the eigenvalues of $\Omega$. Thus a consideration of the roots of (2.3) alone tells one which ones to select in order to construct the minimally inertial specification.

Note also that in most cases, the minimally inertial specification will also minimize $\|\Omega\|$. Thus it not only minimizes the degree to which the value of $\Xi_{t}$ in (A.13) depends on $\Xi_{t-1}$, but also the degree to which $Q\left(L^{-1}\right)$ and $R\left(L^{-1}\right)$ involve projected future, as opposed to current, values of the target variables. This representation expresses the restrictions (A.13) - (A.14) on the admissible evolution of the variables $\left\{\varphi_{t}\right\}$ and $\left\{\tau_{t}\right\}$ in as nearly contemporaneous a form as is possible.

\section{A.3 Assumption 5}

The proof of Proposition 2 relies upon the following additional assumption.

Assumption 5. The vector $B_{2}^{\prime}$ and the vectors $B_{2}^{\prime} \Lambda^{j}$, for $j=1, \ldots, k-1$, are linearly independent of one another. Similarly, the vectors $B_{2}^{\prime} \Lambda^{j}$ for $j=1, \ldots, k$ are linearly independent.

Note that this assumption is violated in particular in the special case in which $B_{2}^{\prime}$ is a left eigenvector of $\Lambda$, or indeed if $B_{2}^{\prime}$ is any linear combination of fewer than $k$ of the left eigenvectors corresponding to non-zero eigenvalues, plus the left eigenvectors corresponding to zero eigenvalues. (Recall that $\operatorname{rank}(\Lambda)=k$, so that there are exactly $k$ non-zero eigenvalues.) It follows that Assumption 5 also requires that $B_{2}^{\prime} g_{i} \neq 0$ for any right eigenvector $g_{i}$ of $\Lambda$ corresponding to a non-zero eigenvalue. Nonetheless, the assumption is clearly satisfied for generic matrices of arbitrary rank, since the elements of $B_{2}$ are not involved in the conditions that define the matrix $\Lambda$. 


\section{A.4 Proof of Proposition 2}

The policy rule (6.6) can be rewritten as

$$
\alpha(L) v_{t}+B_{2}^{\prime} B(L) q_{t}=0
$$

where $q_{t}$ and $v_{t}$ are defined in (6.8) and (6.9) respectively, $\alpha(L)$ is a scalar polynomial of order $k$, and $B(L)$ is a matrix polynomial of order $d=\min \left(k, n_{z}-1\right)$. To apply policy rule (A.38) at all dates $t \geq t_{0}$, one must start with initial conditions $v_{t_{0}-1}, \ldots, v_{t_{0}-k}, q_{t_{0}-1}, \ldots, q_{t_{0}-d}$.

Note that from equations (6.2) and (6.3), the variables $v_{t}$ and $q_{t}$ can determined from the Lagrange multipliers, using the relations

$$
\begin{aligned}
& v_{t}=-B_{2}^{\prime} \Xi_{t} \\
& q_{t}=\Xi_{t}-\Lambda \Xi_{t-1},
\end{aligned}
$$

at all dates $t$. We first show that under Assumption 5, there is a unique sequence of initial Lagrange multipliers $\Xi_{t_{0}-1}, \ldots, \Xi_{t_{0}-d}$ and $\Lambda \Xi_{t_{0}-d-1}$ that satisfies (A.39) - (A.40), and that is consistent with the initial conditions $v_{t_{0}-1}, \ldots, v_{t_{0}-k}, q_{t_{0}-1}, \ldots, q_{t_{0}-d}$ that are part of the specification of $\bar{Z}_{t_{0}}$.

\section{A.4.1 Initial Conditions for Multipliers}

We first note that given the vector $\Lambda \Xi_{t_{0}-d-1}$, and values for $q_{t_{0}-1}, \ldots, q_{t_{0}-d}$, it is possible to solve equations (A.40) uniquely for the vectors $\Xi_{t_{0}-1}, \ldots, \Xi_{t_{0}-d}$. The solutions obtained are of the form

$$
\Xi_{t_{0}-j}=\Lambda^{d+1-j} \Xi_{t_{0}-d-1}+\Delta_{j} q
$$

for $j=1, \ldots, d$, where

$$
q=\left[\begin{array}{c}
q_{t_{0}-1} \\
\vdots \\
q_{t_{0}-d}
\end{array}\right]
$$

and $\Delta_{j}$ is a matrix the elements of which need not be specified. It then remains simply to show that there exists a unique vector $\Lambda \Xi_{t_{0}-d-1}$ such that the solutions (A.41) will also satisfy equations (A.39). 
Substituting the solutions (A.41) into each of the equations (A.39), one obtains a system of equations for the unknown vector $\Lambda \Xi_{t_{0}-d-1}$ that can be written in the form

$$
V=D \Lambda \Xi_{t_{0}-d-1}+F q
$$

where

$$
V=\left[\begin{array}{c}
v_{t_{0}-1} \\
\vdots \\
v_{t_{0}-k}
\end{array}\right], \quad D=\left[\begin{array}{c}
-B_{2}^{\prime} \Lambda^{k-1} \\
\vdots \\
-B_{2}^{\prime}
\end{array}\right],
$$

and again the elements of the matrix $F$ need not be specified. Assumption 5 implies that $D$ is of full rank, i.e., of rank $k$.

Because $\Lambda$ is of rank $k$, the linear space of possible vectors of the form $\Lambda \Xi_{t_{0}-d-1}$ is of dimension $k$. This, together with the fact that $D$ is of rank $k$, guarantees that there is a unique solution $\Lambda \Xi_{t_{0}-d-1}$ to the system of $k$ equations (A.42), regardless of the value of the vector $F q$. It follows that there is exactly one sequence $\Xi_{t_{0}-1}, \ldots, \Xi_{t_{0}-d}$ and one vector $\Lambda \Xi_{t_{0}-d-1}$ that is consistent with any sequence of initial values $v_{t_{0}-1}, \ldots, v_{t_{0}-k}, q_{t_{0}-1}, \ldots, q_{t_{0}-d}$.

\section{A.4.2 Existence of Equilibrium}

Now, given any initial conditions $Z_{t_{0}}, v_{t_{0}-1}, \ldots, v_{t_{0}-k}, q_{t_{0}-1}, \ldots, q_{t_{0}-d}$, we wish to show that there exists a unique bounded solution for $\left\{y_{t}\right\}$ for periods $t \geq t_{0}$ satisfying (2.1) and (6.6) for all $t \geq t_{0}$. We begin by establishing existence of such a solution.

The result of the previous section implies that associated with these initial conditions will be a unique sequence of initial multipliers $\Xi_{t_{0}-1}, \ldots, \Xi_{t_{0}-d}$ and $\Lambda \Xi_{t_{0}-d-1}$, such that (A.39) is satisfied for all $t_{0}-k \leq t \leq t_{0}-1$ and (A.40) is satisfied for all $t_{0}-d \leq t \leq t_{0}-1$. Given the value of $\Xi_{t_{0}-1}$ that is determined in this way, Assumption 1 implies that there is a unique bounded solution $\left\{y_{t}, \xi_{t}, \Xi_{t}\right\}$ satisfying (2.1) and (5.3) for all $t \geq t_{0}$. Proposition 1 then implies that this is also the unique bounded solution to the equation system consisting of (2.1) and (6.2) - (6.4) for all $t \geq t_{0}$. We next wish to show that this solution also satisfies (6.6) for all $t \geq 0$. In that case, we will have established that there exists a solution $\left\{y_{t}\right\}$ that satisfies (2.1) and (6.6), as desired. 
The solution must satisfy

$$
(I-\Lambda L) \Xi_{t}=q_{t}
$$

for each $t \geq t_{0}$, as a consequence of (6.3). The multipliers for the periods prior to $t_{0}$ have been chosen so that (A.43) holds for $t_{0}-d \leq t \leq t_{0}-1$ as well. Thus we can pre-multiply both sides of (A.43) by $B_{2}^{\prime} B(L)$, a lag polynomial of order $d$, and obtain a relation that must hold for all $t \geq t_{0}$. It then follows from (6.5) that

$$
\alpha(L) B_{2}^{\prime} \Xi_{t}=B_{2}^{\prime} B(L) q_{t}
$$

for all $t \geq t_{0}$.

Similarly, the solution must satisfy (A.39) for each $t \geq t_{0}$, as a consequence of (6.2). And the multipliers for the periods prior to $t_{0}$ have been chosen so that (A.39) holds for $t_{0}-k \leq t \leq t_{0}-1$ as well. Thus we can pre-multiply both sides of (A.39) by $\alpha(L)$, a lag polynomial of order $k$, and obtain a relation that must hold for all $t \geq t_{0}$. Substituting this into (A.44), we obtain relation (A.38), which is equivalent to (6.6). Thus the solution shown to exist above must satisfy (6.6) for all $t \geq t_{0}$. It follows that there exists a bounded solution that satisfies both (2.1) and (6.6) for all $t \geq t_{0}$.

\section{A.4.3 Uniqueness of Equilibrium}

Let us next consider the uniqueness of this solution. Suppose that there were some other bounded solution $\left\{y_{t}\right\}$ to (2.1) and (6.6) for all $t \geq t_{0}$ consistent with the given initial conditions $Z_{t_{0}}$ and $v_{t_{0}-1}, \ldots, v_{t_{0}-k}, q_{t_{0}-1}, \ldots, q_{t_{0}-d}$. A unique sequence of multipliers $\Xi_{t_{0}-1}, \ldots, \Xi_{t_{0}-d}$ and $\Lambda \Xi_{t_{0}-d-1}$ consistent with these initial conditions can be constructed as above. Using this initial value for $\Xi_{t_{0}-1}$, one can then construct an associated solution for the multipliers $\left\{\xi_{t}, \Xi_{t}\right\}$ for all $t \geq t_{0}$ using equations (6.3) - (6.4).

We first show that the multipliers constructed in this way must satisfy (6.1) for all $t \geq t_{0}$. By construction, they satisfy (A.43) for all $t \geq t_{0}-d$. Pre-multiplying this by $B_{2}^{\prime} B(L)$, one observes that they must satisfy (A.44) for all $t \geq t_{0}$. Comparing this with (6.6), one can show that

$$
\alpha(L)\left[B_{2}^{\prime} \Xi_{t}+v_{t}\right]=0
$$


for each $t \geq t_{0}$. But since the term in square brackets is zero for each $t_{0}-k \leq t \leq t_{0}-1$ by construction, it follows, by a recursive argument, that it must be zero for each $t \geq t_{0}$ as well. This establishes (6.2).

We next establish that the multipliers constructed in this way are bounded. Let $f_{j}{ }^{\prime}$ and $g_{j}$ be the left and right eigenvectors of the matrix $\Lambda$ corresponding to the eigenvalue $\lambda_{j}$, for $j=1,2, \ldots, n_{z}$, and let these be numbered so that $\left|\lambda_{j}\right|>1$ for $j \leq m$ while $\left|\lambda_{j}\right|<1$ for $j>m$. Let them also be normalized so that $f_{j}{ }^{\prime} g_{j}=1$ for any $j$, while $f_{j}{ }^{\prime} g_{i}=0$ for any $i \neq j$. Then noting that the vectors $g_{j}$ form a basis for $\mathcal{C}^{n_{z}}$, we can express $\Xi_{t}$ as a linear combination of these vectors. Let

$$
\hat{\Xi}_{t} \equiv \Xi_{t}-\sum_{j=1}^{m} g_{j} f_{j}{ }^{\prime} \Xi_{t}
$$

be the projection of $\Xi_{t}$ on the eigenvectors $g_{j}$ with $j>m$, and $\hat{q}_{t}$ be the corresponding projection of $q_{t}$.

It then follows from (A.40) that

$$
\hat{\Xi}_{t}=\Lambda \hat{\Xi}_{t-1}+\hat{q}_{t} .
$$

Because $\hat{\Xi}_{t}$ is a (real-valued) linear combination of the eigenvectors $j>m$, there exists a norm for $\mathcal{R}^{n_{z}}$ in terms of which $\left\|\Lambda \hat{\Xi}_{t-1}\right\| \leq b \cdot\left\|\Xi_{t-1}\right\|$, where $b=\max _{j>m}\left|\lambda_{j}\right|<1$. The triangle inequality together with (A.45) then implies that

$$
\left\|\hat{\Xi}_{t}\right\| \leq b \cdot\left\|\hat{\Xi}_{t-1}\right\|+Q
$$

for all $t \geq t_{0}$, where $Q>0$ is a uniform bound for $\left\|\hat{q}_{t}\right\|$. (A finite bound $Q$ necessarily exists under the hypothesis that $\left\{y_{t}\right\}$ is bounded.) It then follows from recursive application of this inequality that

$$
\left\|\hat{\Xi}_{t}\right\| \leq b \cdot\left\|\hat{\Xi}_{t_{0}-1}\right\|+(1-b)^{-1} Q
$$

for all $t \geq t_{0}$. Thus $\left\{\hat{\Xi}_{t}\right\}$ is necessarily a bounded process.

If $m=0$, the proof that $\left\{\Xi_{t}\right\}$ is bounded is complete. Suppose now instead that $m \geq 1$. Pre-multiplying (A.40) by $f_{j}{ }^{\prime}$ for any $j \leq m$, we obtain a stochastic difference equation for 
the evolution of $f_{j}{ }^{\prime} \Xi_{t}$ that can be solved forward to show that

$$
\lim _{T \rightarrow \infty} \lambda_{j}^{-(T-t)} f_{j}{ }^{\prime} E_{t} \Xi_{T-1}=f_{j}^{\prime} \Xi_{t-1}+\tilde{q}_{t}^{j}
$$

where

$$
\tilde{q}_{t}^{j} \equiv \sum_{T=t+1}^{\infty} \lambda_{j}^{-(T-t)} \mathrm{E}_{t}\left[f_{j}{ }^{\prime} q_{T-1}\right]
$$

is well-defined and bounded because $\left\{y_{t}\right\}$ is bounded and $\left|\lambda_{j}\right|>1$. Hence

$$
\lim _{T \rightarrow \infty}\left\{\mathrm{E}_{t} \Xi_{T-1}-\sum_{j=1}^{m}\left[f_{j} \Xi_{t-1}+\tilde{q}_{t}^{j}\right] \lambda_{j}^{T-t} g_{j}\right\}=\lim _{T \rightarrow \infty} \mathrm{E}_{t} \hat{\Xi}_{T-1}=0 .
$$

Pre-multiplying this by $B_{2}^{\prime}$ and using (A.39), we find that

$$
\lim _{T \rightarrow \infty}\left\{\mathrm{E}_{t} v_{T-1}+\sum_{j=1}^{m}\left[f_{j}{ }^{\prime} \Xi_{t-1}+\tilde{q}_{t}^{j}\right] \lambda_{j}^{T-t} B_{2}^{\prime} g_{j}\right\}=0 .
$$

Because by hypothesis our solution for $\left\{y_{t}\right\}$ is bounded, equation (A.46) can hold only if for each $j=1, \ldots, m$, either $B_{2}^{\prime} g_{j}$ is zero or the term in square brackets is zero. But Assumption 5 requires that $B_{2}^{\prime} g_{j} \neq 0$ for each of these $j$; hence the term in square brackets must be zero in each case. This gives us an expression for $f_{j}{ }^{\prime} \Xi_{t}$ for each $j=1, \ldots, m$, which allows us to write

$$
\Xi_{t}=\hat{\Xi}_{t}-\sum_{j=1}^{m} \tilde{q}_{t+1}^{j} g_{j}
$$

As we have already established that $\hat{\Xi}_{t}$ and each of the $\tilde{q}_{t}^{j}$ are bounded, it follows from this representation that the constructed $\left\{\Xi_{t}\right\}$ is a bounded process. It then follows from (6.4) and the boundedness of $\left\{y_{t}\right\}$ that the constructed process $\left\{\xi_{t}\right\}$ is bounded as well.

We can then apply Proposition 1 to show that the assumed solution $\left\{y_{t}\right\}$ and the constructed multipliers $\left\{\varphi_{t}\right\}$ must satisfy (6.1) for all $t \geq t_{0}$. But this means that we have at least two distinct bounded solutions to the system of equations consisting of $(2.1),(6.1)$ and (6.2), each consistent with the same initial conditions $Z_{t_{0}}$ and $\Xi_{t_{0}-1}$. This is impossible under Assumption 1. Hence there must in fact be a unique bounded solution $\left\{y_{t}\right\}$ to the system consisting of (2.1) and (6.6) under the given initial conditions. 


\section{A.4.4 Optimality}

We have shown in section A.4.1 that there is a unique value of $\Xi_{t_{0}-1}$ consistent with initial conditions $v_{t_{0}-1}, \ldots, v_{t_{0}-k}, q_{t_{0}-1}, \ldots, q_{t_{0}-d}$ and with the conditions (A.39) - (A.40). This solution can be written in the form

$$
\Xi_{t_{0}-1}=g_{0}+g_{Z} \bar{Z}_{t_{0}}
$$

where we we note that $g_{Z}$ places non-zero weight only on elements of $\bar{Z}_{t_{0}}$ that are known at $t_{0}-1$. Furthermore, the unique equilibrium shown above to exist has associated with it a sequence of Lagrange multipliers that satisfy (A.39) - (A.40) in all later periods as well. It follows that these multipliers similarly satisfy

$$
\Xi_{t-1}=g_{0}+g_{Z} \bar{Z}_{t}
$$

for all $t>t_{0}$ as well, where the coefficients are the same as in (A.47).

But then as discussed in section 5 of the text, our bounded solution to (2.1) and (5.3) consistent with $Z_{t_{0}}$ and the initial multipliers $\Xi_{t_{0}-1}$ given by (A.47) involves a plan $\left\{y_{t}\right\}$ that is optimal subject to an additional set of constraints of the form $\tilde{E} z_{t}=\bar{e}$, where

$$
\bar{e}=e_{0}+e_{Z} Z_{t_{0}}+e_{s} s_{t_{0}}+e_{\Xi}\left[g_{0}+g_{Z} \bar{Z}_{t_{0}}\right]
$$

and the coefficients $e_{0}, e_{Z}, e_{s}, e_{\Xi}$ are the ones defined in (5.4). (Their existence is guaranteed by Assumption 1.)

Similarly, the continuation of the plan from any date $t>t_{0}$ onward corresponds to the unique bounded solution to (2.1) and (5.3) consistent with initial conditions $Z_{t}$ and $\Xi_{t-1}$, where $\Xi_{t-1}$ satisfies (A.48). Thus the same reasoning implies that in each period $t>t_{0}$,

$$
\tilde{E} z_{t}=e_{0}+e_{Z} Z_{t}+e_{s} s_{t}+e_{\Xi}\left[g_{0}+g_{Z} \bar{Z}_{t}\right]
$$

Hence the constraint values $\bar{e}$ specified in (A.49) are the same function of $\bar{Z}_{t_{0}}$ and $s_{t_{0}}$ as $\tilde{E} z_{t}$ is of $\bar{Z}_{t}$ and $s_{t}$ according to (A.50). It follows that the plan $\left\{y_{t}\right\}$ is optimal from a timeless perspective, in the sense defined in section 3 of the text. 


\section{A.5 Proof of Proposition 3}

First note that the characteristic polynomial (2.3) is a polynomial of order $\operatorname{rank}(\hat{I})=k+n_{Z}$, where $k \equiv \operatorname{rank}(\tilde{E})$, and thus that admits $k+n_{Z}$ roots $\mu_{i}$, where $i=1, \ldots, k+n_{Z}$. Assumption 7 implies that $(2.3)$ has $(k-m)$ roots such that $\left|\mu_{i}\right| \geq \beta^{-1}$ and $\left(m+n_{Z}\right)$ roots such that $\left|\mu_{i}\right|<\beta^{-1}$, for some $m \geq 1$.

From section A.1, we know that under Assumptions 2 and 3, there exists a square matrix $\Omega$ of dimension $n_{Z}$ for which all of the eigenvalues satisfy $\left|\mu_{i}\right|<\beta^{-1}$. Under Assumptions 2, 3 and 7, the characteristic polynomial (2.3) has therefore $\operatorname{rank}(\hat{I})-n_{Z}=k$ roots that are not among the eigenvalues of $\Omega$, and $(k-m)$ of these roots are such that $\left|\mu_{i}\right| \geq \beta^{-1}$, while $m$ roots are such that $\left|\mu_{i}\right|<\beta^{-1}$. Moreover, we know from Appendix A.1 that under Assumptions 2 and 3 there is a square matrix $\Theta$ of dimension $n_{z}$, and of rank $k$, for which all of the eigenvalues, $\theta_{i}$, for $i=1, \ldots n_{z}$, are either zero or the reciprocal of the roots of (2.3) that are not among the eigenvalues of $\Omega$. Thus the $k$ non-zero eigenvalues of $\Theta$ can be split as follows: $(k-m)$ of these eigenvalues are such that $\left|\theta_{i}^{-1}\right| \geq \beta^{-1}$ and $m$ eigenvalues are such that $\left|\theta_{i}^{-1}\right|<\beta^{-1}$. The remaining $\left(n_{z}-k\right)$ eigenvalues of $\Theta$ are zero.

Let us first consider case (i) of Assumption 2, in which case the matrix $\Theta$ and the associated matrix of eigenvectors $\Psi$ are real. We know from (A.21) that under Assumptions $2-4$, the matrix $\Lambda$ is given by

$$
\Lambda \equiv \beta^{-1} \Psi_{2} \Theta \Psi_{2}^{-1}
$$

where $\Psi_{2}$ is the matrix that is composed of the last $n_{z}$ lines of $\Psi$. Because $\Psi_{2}$ is invertible, it follows that $\Lambda$ and $\beta^{-1} \Theta$ are similar matrices, and so must have the same set of eigenvalues. Thus the eigenvalues of $\Lambda$ are given by $\lambda_{j}=\beta^{-1} \theta_{j}$ for $j=1, \ldots, n_{z}$, or alternatively by $\lambda_{j}=\beta^{-1} \mu_{j}^{-1}$, for $j=1, \ldots, k$, where the $\mu_{j}$ 's are once again the non-zero roots of (2.3) that are not among the eigenvalues of $\Omega$, and $\lambda_{j}=0$ for $j=k+1, \ldots, n_{z}$. Thus the characteristic polynomial of the matrix $\Lambda$ is given by

$$
\operatorname{det}(\Lambda-\lambda I)=\left(\beta^{-1} \mu_{1}^{-1}-\lambda\right) \ldots\left(\beta^{-1} \mu_{k}^{-1}-\lambda\right)(-\lambda)^{n_{z}-k} .
$$

Let us consider the polynomial $\alpha(\chi)=\operatorname{det}(I-\Lambda \chi)$ of order $\operatorname{rank}(\Lambda)=k$, with $k$ roots 
$\chi_{j} \neq 0$, and let these be numbered $j=1, \ldots, k$. Using the previous equation, we have

$$
\begin{aligned}
\alpha(\chi) & =(-\chi) \operatorname{det}\left(\Lambda-\chi^{-1} I\right) \\
& =\left(\beta^{-1} \mu_{1}^{-1}-\chi^{-1}\right) \ldots\left(\beta^{-1} \mu_{k}^{-1}-\chi^{-1}\right)\left(-\chi^{-1}\right)^{n_{z}-k-1}
\end{aligned}
$$

Because the roots $\mu_{j}$ are the non-zero roots of (2.3) that are not among the eigenvalues of $\Omega$, and because $(k-m)$ of these roots are such that $\left|\beta \mu_{j}\right| \geq 1$, and $m$ roots are such that $\left|\beta \mu_{j}\right|<1$, the polynomial $\alpha(\chi)$ has exactly $m$ roots inside the unit circle.

Let us now turn to the case (ii) of Assumption 2, in which the matrices $\Theta$ and $\Psi$ have one complex-valued column. It then follows from (A.36) that $\Lambda$ is given by

$$
\Lambda \equiv \beta^{-1} \bar{\Psi}_{2} \bar{\Theta} \bar{\Psi}_{2}^{-1}
$$

where $\bar{\Psi}_{2}$ is the matrix composed of the last $n_{z}$ lines of $\operatorname{Re} \Psi$, and the matrix $\bar{\Theta}$ is given by

$$
\bar{\Theta} \equiv \operatorname{Re} \Theta=\left[\begin{array}{cc}
\tilde{\Theta} & 0 \\
0 & \operatorname{Re} \mu_{1}^{-1}
\end{array}\right]
$$

where $\tilde{\Theta}$ is the real-valued matrix mentioned in (A.11), and where $\mu_{1}$ (arbitrarily labeled as $\left.\mu_{1}\right)$ is the complex conjugate of the eigenvalue that lies in the lower right corner of $\Omega$ in (A.6). Because of the properties of the latter eigenvalue, and because of Assumption 2, part (ii), we have $\left|\mu_{1}\right|<\beta^{-1}$ and $\left|\operatorname{Re} \mu_{1}^{-1}\right|>\beta$. The $k$ non-zero eigenvalues of $\bar{\Theta}$ can be partitioned as follows: one eigenvalue is equal to $\theta_{1}=\operatorname{Re} \mu_{1}^{-1}$ and satisfies $\left|\theta_{1}\right|>\beta,(m-1)$ eigenvalues are such that $\left|\theta_{j}^{-1}\right|<\beta^{-1}$, and $(k-m)$ eigenvalues are such that $\left|\theta_{j}^{-1}\right| \geq \beta^{-1}$. The eigenvalues of $\Lambda$, i.e., $\lambda_{1}, \ldots, \lambda_{n_{z}}$, are equal to the eigenvalues of $\beta^{-1} \bar{\Theta}$. It follows that $\lambda_{1}=\beta^{-1} \operatorname{Re} \mu_{1}^{-1}, \lambda_{j}=\beta^{-1} \mu_{j}^{-1}$, for $j=2, \ldots, k$, where once again the $\mu_{j}$ 's are the non-zero roots of (2.3) distinct from the root $\mu_{1}$, and that are not among the eigenvalues of $\Omega$, and $\lambda_{j}=0$ for $j=k+1, \ldots, n_{z}$. Thus the characteristic polynomial of the matrix $\Lambda$ is given by

$$
\operatorname{det}(\Lambda-\lambda I)=\left(\beta^{-1} \operatorname{Re} \mu_{1}^{-1}-\lambda\right)\left(\beta^{-1} \mu_{2}^{-1}-\lambda\right) \ldots\left(\beta^{-1} \mu_{k}^{-1}-\lambda\right)(-\lambda)^{n_{z}-k} .
$$

Again, the polynomial $\alpha(\chi)=\operatorname{det}(I-\Lambda \chi)$ admits $k$ roots $\chi_{j} \neq 0$ and can be written as

$$
\alpha(\chi)=\left(\beta^{-1} \operatorname{Re} \mu_{1}^{-1}-\chi^{-1}\right)\left(\beta^{-1} \mu_{2}^{-1}-\chi^{-1}\right) \ldots\left(\beta^{-1} \mu_{k}^{-1}-\chi^{-1}\right)\left(-\chi^{-1}\right)^{n_{z}-k-1} .
$$


Because $\left|\beta^{-1} \operatorname{Re} \mu_{1}^{-1}\right|>1$, and thus $\left|\beta^{-1} \operatorname{Re} \mu_{1}^{-1}\right|^{-1}<1$, and because $(m-1)$ roots $\mu_{j}$ among the roots $\mu_{2}, \ldots, \mu_{k}$ are such that $\left|\beta \mu_{j}\right|<1$ and that the remaining $(k-m)$ are such that $\left|\beta \mu_{j}\right| \geq 1$, the polynomial $\alpha(\chi)$ has exactly $m$ roots inside the unit circle.

We know from the text that under Assumption 7 the polynomial $\alpha(L)$ reduces to $1-$ $\gamma(L) L$, and it is the lag polynomial that enters the optimal policy rule (7.1), when $\lambda_{i}>0$. Thus under Assumptions $1-7$, with $\lambda_{i}>0$ - and for both cases (i) and (ii) of Proposition 2 - the optimal interest-rate rule (7.1) necessarily involves weights on lagged interest rates that are large enough for the lag polynomial to have $m$ zeros inside the unit circle.

Let us now factor the lag polynomial $\alpha(L)$ as

$$
\alpha(L)=\alpha_{1}(L) \alpha_{2}(L)
$$

where $\alpha_{1}(L)$ is a polynomial of order $m$ with all its zeros, $\chi_{1}, \ldots, \chi_{m}$, inside the unit circle and $\alpha_{2}(L)$ is a polynomial of order $k-m$ with all of its zeros outside the unit circle. Note that $\alpha_{1}(L)$ can be expressed as

$$
\begin{aligned}
\alpha_{1}(L) & =\alpha_{0}\left(\chi_{1}-L\right) \ldots\left(\chi_{m}-L\right) \\
& =L^{m} \alpha_{0}\left(\chi_{1} L^{-1}-1\right) \ldots\left(\chi_{m} L^{-1}-1\right) \\
& \equiv L^{m} \tilde{\alpha}_{1}\left(L^{-1}\right),
\end{aligned}
$$

where $\alpha_{0} \neq 0$, and

$$
\tilde{\alpha}_{1}\left(L^{-1}\right)=(-1)^{m} \alpha_{0}\left(1-\chi_{1} L^{-1}\right) \ldots\left(1-\chi_{m} L^{-1}\right) .
$$

Using this, and assuming again that $\lambda_{i}>0$, we can rewrite the optimal policy rule (7.1) as

$$
\lambda_{i} \alpha(L)\left(i_{t}-i^{*}\right)=-B_{2}^{\prime} B(L) \mathrm{E}_{t}\left[Q\left(L^{-1}\right) \hat{T}^{\prime} \hat{W}\left(\hat{\tau}_{t}-\hat{\tau}^{*}\right)\right]
$$

or equivalently as

$$
\lambda_{i} L^{m} \tilde{\alpha}_{1}\left(L^{-1}\right) \alpha_{2}(L)\left(i_{t}-i^{*}\right)=-B_{2}^{\prime} B(L) \mathrm{E}_{t}\left[Q\left(L^{-1}\right) \hat{T}^{\prime} \hat{W}\left(\hat{\tau}_{t}-\hat{\tau}^{*}\right)\right] .
$$


Because all of the zeros $\chi_{1}, \ldots, \chi_{m}$ are inside the unit circle, the polynomial $\tilde{\alpha}_{1}\left(L^{-1}\right)$ is invertible, and the above equation is equivalent to

$$
\lambda_{i} \alpha_{2}(L)\left(i_{t-m}-i^{*}\right)=-\mathrm{E}_{t}\left\{\tilde{\alpha}_{1}\left(L^{-1}\right)^{-1} B_{2}^{\prime} B(L) \mathrm{E}_{t}\left[Q\left(L^{-1}\right) \hat{T}^{\prime} \hat{W}\left(\hat{\tau}_{t}-\hat{\tau}^{*}\right)\right]\right\},
$$

in the sense that bounded processes $\left\{i_{t}\right\}$ and $\left\{\hat{\tau}_{t}\right\}$ satisfy one relation for all $t \geq t_{0}$ if and only if they satisfy the other for all $t \geq t_{0}$. Thus rules (7.1) and (7.5) are equivalent in this sense. 